\title{
Bargaining with Hard Evidence
}

DOI:

10.1111/ecoj.12632

\section{Document Version}

Accepted author manuscript

Link to publication record in Manchester Research Explorer

\section{Citation for published version (APA):}

Wallace, C., \& Eso, P. (2019). Bargaining with Hard Evidence. The Economic Journal, 129(621), 2039-2063. https://doi.org/10.1111/ecoj.12632

\section{Published in:}

The Economic Journal

\section{Citing this paper}

Please note that where the full-text provided on Manchester Research Explorer is the Author Accepted Manuscript or Proof version this may differ from the final Published version. If citing, it is advised that you check and use the publisher's definitive version.

\section{General rights}

Copyright and moral rights for the publications made accessible in the Research Explorer are retained by the authors and/or other copyright owners and it is a condition of accessing publications that users recognise and abide by the legal requirements associated with these rights.

\section{Takedown policy}

If you believe that this document breaches copyright please refer to the University of Manchester's Takedown Procedures [http://man.ac.uk/04Y6Bo] or contact uml.scholarlycommunications@manchester.ac.uk providing relevant details, so we can investigate your claim.

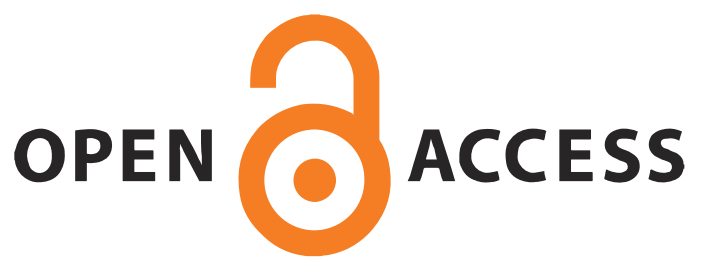




\title{
Bargaining with Hard Evidence
}

\author{
Péter Eső \\ University of Oxford \\ peter.esodeconomics.ox.ac.uk \\ Chris Wallace ${ }^{\dagger}$ \\ University of Manchester \\ christopher.wallace@manchester.co.uk
}

May 23, 2018

\begin{abstract}
This paper studies how the presence of concealable hard evidence affects the timing of agreement and the size and distribution of surplus in bargaining. A buyer and a seller receive randomly-arriving, verifiable, but concealable evidence about the value of a tradable good. Each party discloses individually favourable information but conceals signals that benefit the other side, giving rise to mutual suspicion; the seller repeatedly posts prices valid for one period. In the leading case of interest with a finite horizon and sufficiently patient players, the equilibrium is characterized by an interval of skimming (a sequence of prices acceptable only to a buyer who has learnt that the good's value is high) concluded by a single settlement period in which agreement is reached for sure. The length of delay until agreement and the corresponding efficiency loss are decreasing in the time horizon and in the abilities of the trading parties to identify the good's value, but increasing in impatience. An arbitrarily long time horizon leads to immediate agreement if the parties are even slightly impatient and interaction is frequent enough; if the parties are perfectly patient there is no trade without evidence disclosure.
\end{abstract}

JEL Classification Codes. C78, D82, D83. Keywords. Bargaining, hard evidence, verifiable information, information disclosure, skimming, settling, delay.

\section{INTRODUCTION}

Most game-theoretic models of bargaining abstract away from the role of the discovery and disclosure of verifiable evidence. Instead, the focus is often on the implications of the parties' bargaining power, as captured by the order and frequency of offers, their risk attitudes, their impatience, or their access to unverifiable private information. ${ }^{1}$ The goal of this paper is to capture, within a standard dynamic bargaining game, a player's ability to discover and prove facts concerning the value or size of the surplus, and analyse its effects on the timing, efficiency, and equity of bargaining outcomes.

Bargainers have an incentive to disclose information favourable to themselves while concealing information beneficial to the other party. Naturally, therefore, uninformed (and

\footnotetext{
Acknowledgements. We thank the editor and two referees for their comments. We also thank seminar participants at Oxford, Leicester, and Manchester amongst (many) others for their questions and suggestions. Special thanks to Simon Board, Vincenzo Denicolò, Johannes Hörner, María Sáez Martí, David P. Myatt, Thomas Noe, Joel Sobel, and Eyal Winter for helpful discussions and advice. This version replaces an earlier, entitled "Persuasion and Pricing: Dynamic Trading with Hard Evidence".

†Corresponding Author. Department of Economics, University of Manchester, Manchester M13 9PL, UK.

${ }^{1}$ For a textbook treatment of bargaining theory, see Muthoo (1999). Kennan and Wilson (1993) and Ausubel, Cramton, and Deneckere (2002) provide surveys of bargaining games with incomplete information.
} 
hence silent) players will be suspicious of each other, making agreement more difficult to reach. On the other hand, delaying agreement longer gives players more time to discover and disclose information in their own self-interests. A key contribution of the paper is to show that this fundamental tension is resolved in an equilibrium featuring delay in the absence of disclosure, as players wait in the hope of discovering favourable information, followed by a single settlement period in which agreement is reached for sure. The length of time that uninformed players must wait until agreement occurs is determined by a variety of important factors: the skill of the players in discovering the value of the surplus, the players' patience, and the window of opportunity in which the players can trade, to name a few. A second contribution of this paper is to provide testable comparative statics in these terms. For instance, bargainers who are more likely to be able to generate verifiable evidence settle earlier; so do more patient parties, or those with a longer window of opportunity in which to conclude their negotiations.

In general terms, the model is motivated by the importance of verifiable, perhaps documentary, evidence in practical bargaining situations such as those found in labour market negotiations or settlement talks in commercial disputes. Everyday experience as well as written accounts of, and advice for, real-life negotiations often focus on the parties' ability to generate and disclose persuasive, hard information. ${ }^{2}$ Verifiable information disclosure is at the heart of the large literature on quality disclosure and certification, typically with one side of the market able to prove or certify the quality of its product. ${ }^{3}$

The paper draws some of its inspiration from literary accounts of negotiations, where the emphasis is often on strategic evidence disclosure rather than the mechanics of offers, counteroffers, or deadlines. For example, the story by Dahl (1979) entitled Parson's Pleasure describes the adventures of a fictional but cunning London antiques dealer. On Sundays he dresses up as a vicar (to disguise his expertise) and knocks on the doors of countryside homes under various pretenses; once he is in, he looks around for antiques to buy on the cheap. The heart of the story is a negotiation over a Chippendale commode. The conman/antiquarian tries to convince his uneducated hosts that the cupboard is a worthless fake, while they unwittingly produce irrefutable evidence of its provenance and exquisite value. In the end, he prevails: he pays twenty pounds for the item, and goes to collect his car. The punchline of Dahl's story is that in the end, the fake vicar's persuasive arguments work too well. While he is away the hosts helpfully turn the commode into what he had convinced them it was worth: a pile of firewood. For a game theorist, what's remarkable is that most of the bargaining process concerns information transmission, whereas the price is set rather quickly. In fiction and in reality, the secret of success in negotiation is arguably the production and disclosure of credible evidence.

\footnotetext{
${ }^{2}$ In a practical book on negotiations Fisher and Ury (1982) suggest that parties emphasize objective criteria such as focusing on the value of accessories when bargaining over a used car (Chapter 5). Another popular book on bargaining, Raiffa (1982), discusses the selective disclosure of information in Chapter 9 (p. 127), entitled "Advice for Negotiators". The same book reports a survey of banking executives on the characteristics of an effective negotiator (pp. 121-122): it ranks Ability to Persuade 8th out of 34, one place ahead of Patience. ${ }^{3}$ For a comprehensive survey of both theory and empirical work in this field, see Dranove and Jin (2010).
} 
Nevertheless, bargaining theory to date has been largely silent on the potential effects of the presence of such evidence (a review of the related literature is postponed to Section 6). For now, to make matters concrete, consider the following familiar scenario from the junior academic job market, which most accurately motivates the model that follows.

A job-market candidate (he) and a department chair (she) are bargaining over his starting salary. The department wants to reach agreement before the end of the job-market round; else the position remains vacant for a year and the department has to pay for temporary teaching cover. Equally, if no agreement is reached in the relevant time-frame, the candidate has to return to the market the following academic year and try his luck again.

The candidate has a paper in submission at a top general-interest journal. A successful outcome for this paper provides hard evidence of the candidate's quality and radically transforms his market prospects. He could, if he were to receive a response from the journal's editor during the bargaining process, disclose the contents of the letter to the department chair. Clearly, it could be in the interests of the candidate to disclose if the news was good; in the event of a rejection, he might well choose not to mention it. Critically, however, there is no way for the candidate to prove he has not received a response.

The department chair is likely well connected in the profession, and may very well know one or more of the editors or referees involved. She may therefore, independently from the candidate, also receive word of the paper's progress. She may hesitate to reveal a successful outcome for the paper to the candidate, thereby raising the salary, research budget, or other perks required to attract him. Nevertheless, the chair would accede to such demands made by the candidate, if he proved-or if the chair otherwise learned-that the paper had been accepted. On the other hand, she might wish to disclose the evidence in the event the paper is to be rejected, or she may simply wish to walk away. Crucially, again, the department chair cannot prove that she has not obtained any evidence.

Of course, given the length of time typically involved in the editorial process, it is entirely possible that no evidence arrives with either the candidate or the department chair throughout the entire bargaining process. Can agreement be reached within the relevant time-frame, even for a candidate concealing a rejection? How does the possibility of hardevidence disclosure affect the timing of agreement, the price at which they agree (if they do), and, therefore, the surplus the parties are able to extract?

An alternative motivational setting is provided by real-estate negotiations. A buyer and seller bargaining over the price of a residential property may be able to generate verifiable evidence of its quality. In particular, a buyer may appoint a surveyor whose report she is at liberty to disclose to the seller. Of course, a buyer is unlikely to reveal the surveyor's report (and is under no obligation to do so) if it proves the house is in good order; rather it is in her interests to reveal the report whenever it uncovers some defect forcing the seller to reduce his price. On the other hand, the seller may (or may not) have access to documentary evidence of various works done, engineers' reports and so on, which he can disclose to the buyer should he so wish. Importantly, neither buyer nor seller is obliged to disclose such evidence, and only has an incentive to do so when it is in their own favour. Moreover, 
neither can demonstrate to the other party that they do not have such reports in their possession. Similar questions arise: how does the possibility of hard-evidence disclosure affect whether and when agreement is reached, the price agreed, and the surplus accruing to the two parties?

The model developed in Section 2 captures many of the features of the above scenarios. Departing from the specific job-market or real-estate negotiation settings, a more abstract bargaining game is presented using the language of a buyer and seller (who, in the first story above, would be the department chair and the candidate respectively) trading a single indivisible good. Over time, each player may be able to generate verifiable but concealable evidence about the good's value, while the seller is making repeated take-it-or-leave-it offers to the buyer. The equilibrium is characterized in Section 3. It determines the time and price of settlement (agreement in the absence of either side proving the good's value to the other), as well as the prices set by the seller before and, off the equilibrium path, after the time of settlement. The fundamental tension is that both parties suspect the other of concealing disadvantageous evidence from the first moment they meet. However, they are also aware that any delay gives them more time to find and disclose evidence to their own advantage, while simultaneously reducing the surplus that can be shared, perhaps because of discounting or, with a finite horizon, a deadline.

In the leading case of interest analysed in Section 4, with a finite time horizon and sufficiently patient players, equilibrium play is characterized by a finite delay absent disclosure before sure settlement. After settlement off the equilibrium path the players would once again wait until the final period in which (again, absent disclosure) no trade would take place. The equilibrium constructed in the key Proposition 1 has a striking "wait-settlewait" structure.

The rough intuition is as follows. At the first feasible trading date, by assumption, both parties may already be concealing information beneficial for the other side. Hence, owing to adverse selection, a genuinely uninformed but 'suspicious' seller is unable to make an offer that a similarly uninformed and suspicious buyer would be willing to accept: both would rather wait for the arrival of more information. Over time, if the seller discloses nothing, the uninformed buyer becomes increasingly pessimistic about the good's value. If the time horizon is long enough, the uninformed buyer's beliefs when the seller makes no disclosure will eventually lie below the value the seller attaches to retaining the good. No sale would be made in the very last period in such a circumstance. Anticipating this in earlier periods, and running out of time to discover and disclose hard evidence, an uninformed seller fears that no agreement will ever be reached. Even with little or no discounting, then, he would prefer to offer a discrete price cut in order to entice trade. In equilibrium the discount is offered gradually over time, in the form of delay, unless either party discovers that the good is valuable, until an endogenous settlement date is reached.

In the finite-horizon case the above-mentioned finite delay is shortened, and the corresponding efficiency loss decreased, by a greater ability of either party to identify the good's value. Perhaps surprisingly the delay is also shortened by a longer time horizon or a greater degree of patience. This is because any of these exogenous changes increases the 
seller's continuation payoff from 'skimming' (setting a high price that only a buyer privately informed of the good's high value is willing to pay). At the time of settlement the price must compensate the seller for this alternative continuation payoff. However, a higher settlement price requires earlier settlement because the uninformed and increasingly suspicious buyer's willingness to pay is falling over time.

In contrast to the positive but limited delay in the finite-horizon case, under an arbitrarily long time horizon settlement may occur immediately, or never. The analysis of Section 5 demonstrates that the former occurs when offers are made arbitrarily frequently and with any positive degree of impatience, whereas the latter occurs when the parties are infinitely patient. If the trading partners do not discount future payoffs then they may as well wait until the true value of the good is discovered and revealed, so that trade can take place at the 'fair' price. Hence they never settle. If, however, the players are even slightly impatient then the seller is willing to agree to a price cut, but the discount the seller is willing to offer tends to zero as the time horizon grows unboundedly.

\section{A BARGAINING GAME WITH HARD EVIDENCE}

This section presents the general model and describes the relevant equilibrium concept.

A seller $(S$, he) has an indivisible good for sale to a single buyer $(B$, she). The state of the world is $\omega \in\{H, L\}$. If $\omega=H$ then the good's monetary value is 1 for $B$ and $v \in(0,1)$ for $S$; if $\omega=L$ then it is 0 for both players. $v$ is commonly known, whereas the realization of $\omega$ is initially unknown to both parties; they have a common prior, $\sigma=\operatorname{Pr}[\omega=H] \in(0,1)$.

Information Structure and Timing of Actions. Publicly-observable physical time is indexed by $t \in\{\Delta, 2 \Delta, \ldots\}$. Discrete time makes the order of moves clear and the players' strategies well-defined. $\Delta>0$ is thought of as small throughout; the case of arbitrarily frequent interaction (discrete time with $\Delta \rightarrow 0$ ) will be studied for being inherently interesting and analytically amenable. ${ }^{4}$

At the beginning of each period, or equivalently during the preceding $\Delta$ amount of time, $S$ learns the state of the world with probability $r_{S} \Delta$, and otherwise remains uninformed. Independently, $B$ learns the state with probability $r_{B} \Delta$ and otherwise remains uninformed. Directly following this, each player may either verifiably disclose or conceal knowing the state; however, a lack of information about the state cannot be proved.

Formally, at the beginning of each period $t, S$ observes $s_{t} \in\{L, H, \emptyset\}$ and, simultaneously and independently, $B$ observes $b_{t} \in\{L, H, \emptyset\}$. An "empty" observation, denoted $\emptyset$, for either player is taken to mean that no hard evidence about $\omega$ is obtained. Conversely, nonempty observations are perfectly revealing of the state $\omega$, with

$$
\operatorname{Pr}\left[s_{t}=\omega\right]=1-\operatorname{Pr}\left[s_{t}=\emptyset\right]=r_{S} \Delta \quad \text { and } \quad \operatorname{Pr}\left[b_{t}=\omega\right]=1-\operatorname{Pr}\left[b_{t}=\emptyset\right]=r_{B} \Delta .
$$

\footnotetext{
${ }^{4}$ The limit is made explicit from the latter half of Section 3 onwards. At this point, it is worth noting that $\Delta \rightarrow 0$ is not essential for much of the analysis: it would be straightforward, if algebraically cumbersome, to reformulate the results for a fixed period length throughout.
} 
$s_{t}, b_{t} \in\{H, L\}$ are verifiable, but $s_{t}=\emptyset$ and $b_{t}=\emptyset$ are not. When a nonempty $s_{t}$ or $b_{t}$ is disclosed at any $t^{\prime} \geq t, \omega$ becomes commonly known to the players. ${ }^{5}$ The idea is that $S$ (respectively, $B$ ) has the ability to generate irrefutable evidence regarding the good's value at a rate $r_{S}$ (respectively, $r_{B}$ ) per unit time, but when a player finds such evidence (for instance, a certificate of the good's provenance) he or she is able to conceal it.

$S$ and $B$ may trade at the end of period $t$ provided it falls within the exogenously given time interval $[\underline{t}, \bar{t}] \subseteq[0, \infty]$. Let $\mathbb{T} \equiv[\underline{t}, \bar{t}] \cap\{\Delta, 2 \Delta, \ldots\}$ denote the set of trading periods and $T \equiv \max \mathbb{T}$ the last such period (which coincides with $\bar{t}$ unless $\bar{t}$ is not divisible by $\Delta$ ). Note that $\mathbb{T}$ does not include 0 but is otherwise general. At each $t \in \mathbb{T}$ the players first may simultaneously disclose any evidence obtained at or before $t$, then $S$ makes a take-it-or-leave-it price offer, $p_{t}$. If $B$ accepts she pays $p_{t}$ and realizes the good's value; if she rejects play continues in the next period. If the offer is rejected in the final period $S$ keeps the good, realizing the salvage value $v$ when $\omega=H$, or nothing when $\omega=L$; either way, $B$ receives nothing. Both players are risk neutral with respect to monetary gains, and discount payoffs at a rate $\rho \geq 0$ per unit time.

Note that $S$ and $B$ may obtain information before they start bargaining: even if $\underline{t}=0$, the first opportunity to trade arises at the end of $t=\Delta$. This can create a gap between the players' initial estimates regarding the good's value. To see this, suppose $S$ is believed to conceal "bad" signals and $B$ to hide "good" ones. This is indeed in each player's self interest, as $S$ wants to sell high and $B$ to buy low. Then a genuinely uninformed $S$ will be more optimistic about $\omega=H$ than a genuinely uninformed $B$ by the time the first offer is made. Moreover, the larger is $\underline{t}$, the larger will be the gap between the players' initial beliefs. That the trading window $\mathbb{T}$ might start after several chances for the players to gather evidence is an intended feature of the model: while, for the most part, it is without loss to assume $\underline{t}=0$, allowing $\underline{t}>0$ to vary in principle allows a consideration of how larger differences in the player's pre-bargaining beliefs affect equilibrium outcomes. ${ }^{6}$

Solution Concept. Equilibrium henceforth refers to pure-strategy perfect Bayesian equilibrium as defined in Fudenberg and Tirole (1991, Definition 8.2), suitably extended to the present context with a continuum of actions. Additionally, the following two refinements on out-of-equilibrium beliefs will be imposed. First, if $S$ makes an out-of-equilibrium price offer, then $B$ 's beliefs are required to satisfy the Intuitive Criterion. That is, $B$ must put zero weight on a "type" of $S$ that could not possibly gain from the deviation for any sequentially rational response of $B$ (types are described formally below). Second, if $B$ strays from equilibrium by rejecting a price that she should have accepted for sure, irrespective of her private information, then $S$ continues to believe that $B$ is uninformed. As will be clear from the analysis, this is a sensible and intuitively appealing assumption. ${ }^{7}$

\footnotetext{
${ }^{5}$ Note players cannot obtain contradictory signals (e.g., $s_{t}=H$ and $b_{t^{\prime}}=L$ ), and this is commonly known. The assumption that signals (when obtained) are perfect is made for analytic tractability: considering similar models with imperfect hard evidence would be an interesting topic for future research.

${ }^{6} \mathrm{All}$ of this is conceptually distinct from commonly known but different priors. Incorporating different priors in this model is an interesting direction for future work.

${ }^{7}$ In particular, if $S$ makes an offer that an uninformed buyer is just indifferent between accepting and rejecting then a buyer who has previously observed $\omega=H$ has a strict incentive to accept.
} 
If $S$ observes $s_{t}=H$ then it is optimal for him to disclose this fact and charge $p_{t}=1$, which ought to be accepted by $B$ without delay. ${ }^{8}$ A mirror-image assumption is made for the other side of the market: if $B$ observes $b_{t}=L$ then she will disclose it immediately, inducing $S$ to set $p_{t}=0$ for subsequent acceptance and trade. This can be thought of as an indifference-breaking condition, as both parties earn zero surplus whether or not they trade at zero price in state $\omega=L$. An alternative assumption that works equally well is that $B$ "walks away" from the relationship immediately upon learning that $\omega=L$, perhaps because she has other, more productive things to do (outside the model).

As a result of this indifference-breaking condition and the other observations made in the previous paragraph, at any $t \in \mathbb{T}$ and absent prior disclosure, there may be two types only of $B$ present: the uninformed buyer, labelled $B_{\emptyset}$, and an informed buyer who knows the state is high, labelled $B_{H}$. Equally, there may be two types only of $S$ present: an uninformed $S_{\emptyset}$ and an informed seller who knows the state is low, $S_{L}$.

The facts that self-serving signals are immediately disclosed (ending the game) whereas self-defeating ones are concealed (to keep the other player guessing) are intended, key features of the model. ${ }^{9}$ The seller's objective is to find an optimal price-discrimination strategy knowing that he faces a buyer who is either uninformed or, if informed, concealing an $H$-signal, while being aware that an uninformed $B$ suspects him of potentially concealing an $L$-signal. Since $S$ knows that, at any $t \in \mathbb{T}$, he faces either an uninformed buyer $\left(B_{\emptyset}\right)$ or one who knows the good is valuable $\left(B_{H}\right)$, he must either charge a relatively high price that only the latter type would be prepared to accept, called "skimming", or a lower price that both buyer types would accept for sure, called "settling". 10

The unique equilibrium satisfying the above properties is constructed in the next section.

\section{EQUILIBRIUM CONSTRUCTION}

In this section an equilibrium is characterized in which at each $t \in \mathbb{T}$ the parties disclose all self-serving signals received at or before $t$ and, conditional on such disclosure, immediately trade at a price equal to $B^{\prime}$ s valuation. If no disclosure is made then both types $S_{\emptyset}$ and $S_{L}$ of the seller either skim, by offering a price $p_{t}$ acceptable only to $B_{H}$, or settle, by offering a price $q_{t}$ acceptable to both $B_{\emptyset}$ and $B_{H}$. After an out-of-equilibrium price proposal $B^{\prime}$ s beliefs satisfy the Intuitive Criterion. In case, out of equilibrium, a settlement price is rejected, then $S$ believes $B$ is uninformed and makes no inference regarding the state. Self-defeating signals are concealed in equilibrium.

\footnotetext{
${ }^{8}$ Immediate disclosure of $s_{t}=H$ is strictly optimal for $S$ if $\rho>0$, but for continuity the same is assumed in this event for $\rho=0$ as well. The fact that after such disclosure $B$ accepts $p_{t}=1$ right away follows from the usual reasoning that otherwise $S$ could slightly lower the price to ensure acceptance.

${ }^{9}$ The first part of this statement is justified in Footnote 8. For the second part note that disclosing $b_{t}=H$ results in immediate trade at price 1 , so $B$ is either strictly worse off revealing $H$ (if a lower price is expected in any subsequent period; "settling", see below) or at best indifferent (if $S$ intends to charge a high price up to and including $T$; "skimming", see below). The very same argument applies for $S$ concealing $s_{t}=L$.

${ }^{10}$ Of course, $S$ could simply "delay" as well, perhaps by offering a price so high no type of $B$ could accept. As will be shown in the proof of Proposition 1 (see the appendix), when $r_{B}>0$, so that the buyer may be informed, skimming is strictly better than delay if $\rho>0$.
} 
Proposition 1 establishes the existence and uniqueness of an equilibrium with these properties. The equilibrium either prescribes skimming in all periods or, for instance, when the horizon is sufficiently long but finite, involves a final period of settlement $m$, after which, off the equilibrium path, $S$ would again skim in all periods. Before reaching $m$, in the absence of signal disclosure by either party, the seller skims in all periods provided the time horizon is finite and the players are sufficiently patient.

Both on and off equilibrium path, $S_{L}$ pools with $S_{\emptyset}$ when offering skimming and settling prices. This is not assumed: there is no equilibrium in which $S_{L}$ separates from $S_{\emptyset}{ }^{11}$

Equilibrium Characterization. In order to derive the last settlement date, $m$, two quantities are defined: an uninformed buyer's willingness to pay, $\beta_{t}$, and an uninformed seller's expected payoff from skimming at $t$ and in all subsequent periods, $V_{t}$.

Absent any disclosure by $S$ at or before $t$, the good's expected value for a genuinely uninformed $B$ may be computed using Bayes' rule. For all $t \in\{\Delta, 2 \Delta, \ldots\}$,

$$
\beta_{t}=\operatorname{Pr}\left[\omega=H \mid B_{\emptyset}, \forall \tau \leq t: s_{\tau} \in\{\emptyset, L\}\right]=\frac{\sigma\left(1-r_{S} \Delta\right)^{t / \Delta}}{\sigma\left(1-r_{S} \Delta\right)^{t / \Delta}+1-\sigma} .
$$

$\beta_{t}$ is strictly decreasing in $t$ : an uninformed buyer loses confidence in the good's value as $S$ remains silent, that is, he does not prove $\omega=H$. For $B$ it becomes increasingly likely that $S$ is in fact concealing a low signal. As $t \rightarrow \infty$, this expected valuation tends to zero. Of course, as time passes, it also becomes less likely that $B$ remains uninformed.

The seller believes that his price proposal is only ever rejected by buyer-type $B_{\emptyset}$, on or off the equilibrium path, regardless of whether $S$ has skimmed or attempted to settle. This is a consequence of the second refinement discussed in Section 2. If the offer was intended for informed buyers only (skimming), then it is an equilibrium requirement for the seller to have such beliefs. If the offer was intended for all buyer-types (settling), and is rejected, the seller's beliefs are arbitrary in a perfect Bayesian equilibrium. Here the refinement bites. As will be seen, the optimal settling price makes the uninformed buyer just indifferent between accepting and rejecting; whereas it gives positive surplus to the informed buyer who knows $\omega=H$. Thus, loosely, the latter is less likely to deviate from the prescribed equilibrium action. It is in this sense that the refinement is reasonable.

Hence, right before the beginning of period $t$, i.e., before $s_{t}$ and $b_{t}$ are observed, $S$ believes $B$ is uninformed. Therefore, if the seller remains uninformed at $t$ and the buyer makes no disclosure either, then $S_{\emptyset}$ believes the state is $\omega=H$ in period $t$ with probability

$$
\hat{\sigma}=\operatorname{Pr}\left[\omega=H \mid S_{\emptyset}, b_{t} \in\{\emptyset, H\}, \forall \tau<t: b_{\tau}=\emptyset\right]=\frac{\sigma}{\sigma+(1-\sigma)\left(1-r_{B} \Delta\right)} .
$$

\footnotetext{
${ }^{11}$ If a settling price is offered in some future period, $S_{L}$ has a strict incentive to deviate from separation to mimic $S_{\emptyset}$ and thus, with positive probability, receive a non-zero payoff once settlement is reached. This is because separation must result in a zero payoff for $S_{L}$. On the other hand, if no settling price is ever to be offered in the future and $S_{L}$ separated from $S_{\emptyset}$, then $B_{\emptyset}$ would accept a settling price $q_{t}=\sigma$ from $S_{\emptyset}$. This would be preferred to skimming by $S_{\emptyset}$ since the probability of receiving 1 from skimming is $r_{B} \Delta$, and $r_{B} \Delta<\sigma$ for $\Delta>0$ sufficiently small. However, there can be no separating equilibrium where $S_{\emptyset}$ offers a settlement price $\sigma$ in the absence of future settlement since, again, $S_{L}$ would wish to mimic.
} 
Note that $\hat{\sigma}$ does not depend on $t$ : it is a constant that exceeds $\sigma$ but converges to it as $\Delta \rightarrow 0$. Intuitively, $\hat{\sigma}>\sigma$ as $B$ might have learned $\omega=H$ exactly at $t$, but the chance of that happening is infinitesimal as the period length becomes arbitrarily small.

In the final trading period, at $T=\max \mathbb{T}$, the seller may skim using $p_{T}=1$ as $B_{H}$ has zero continuation value from rejecting the final offer. The expected payoff of $S_{\emptyset}$ from skimming at $T$ is

$$
V_{T}=\hat{\sigma}\left[r_{B} \Delta+\left(1-r_{B} \Delta\right) v\right]
$$

because, conditional on $\omega=H$ (i.e., with probability $\hat{\sigma}$ ), $S_{\emptyset}$ sells to $B_{H}$ at price 1 with probability $r_{B} \Delta$ and otherwise keeps the good that is worth $v$ to him. On the other hand, if $\omega=L$ then the seller gets no payoff from skimming in the last period. For any $t<T$, if the seller plans to skim with price 1 in all subsequent periods then he can skim at $t$ using price $p_{t}=1$ as well, and his expected payoff from doing so is $V_{t}$ satisfying

$$
V_{t}=\hat{\sigma}\left[r_{B} \Delta+\frac{\left(1-r_{B} \Delta\right) r_{S} \Delta}{1+\rho \Delta}\right]+\frac{\left(1-r_{B} \Delta\right)\left(1-r_{S} \Delta\right)}{1+\rho \Delta} V_{t+\Delta} .
$$

If the state is high, which has probability $\hat{\sigma}$ from the perspective of $S_{\emptyset}$, then $B$ accepts the skimming price with probability $r_{B} \Delta$. Else, if she is not of type $B_{H}$ already, then $S_{\emptyset}$ may become informed of $\omega=H$ within the next period and sell the good at price 1 with a oneperiod delay. If either player learns $\omega=L$ within the next period then the seller's payoff is zero. If neither player learns the state by the next period, then $S_{\emptyset}$ will get a continuation payoff $V_{t+\Delta}$ with $\Delta$ delay. The expressions in (2) and (3) uniquely define $V_{t}$.

So, if $S_{\emptyset}$ skims at $t$ using $p_{t}=1$, anticipating that, absent disclosure, he will skim in all future periods as well, then his expected payoff is $V_{t}$. Alternatively, he can settle with price and sure payoff $q_{t}=\beta_{t}$, which is acceptable to both buyer types. Hence, provided he will skim in all future periods, $S_{\emptyset}$ prefers skimming to settling at $t$ if and only if $V_{t}>\beta_{t}$. This condition holds in the final period if the time horizon is sufficiently long because $V_{T}$ is a positive constant irrespective of $T$, whereas $\beta_{t}$ converges to zero as $t \rightarrow \infty$.

If $V_{t}>\beta_{t}$ for all $t \in \mathbb{T}$ then let $m=0$, otherwise define

$$
m=\max \left\{t \in \mathbb{T} \mid V_{t} \leq \beta_{t}\right\}
$$

This is the final moment of settlement: the last time of certain trade without disclosure. By definition $V_{t}>\beta_{t}$ holds in all periods $t>m$, hence $S_{\emptyset}$ skims using $p_{t}=1$ provided neither player reveals the state at or before $t .{ }^{12}$ Note that $S_{L}$ also skims at all $t>m$ even though he expects zero payoff by doing so: a deviation, from which only $S_{L}$ could gain, would reveal his type by the Intuitive Criterion and result in zero payoff as well. This is where the first refinement discussed in Section 2 is used. The equilibrium offers from $S$ are constructed to be optimal for $S_{\emptyset}$. Thus, regardless of $B^{\prime}$ s decision following a non-equilibrium price

\footnotetext{
${ }^{12}$ The definition in (4) is silent on how many times $V_{t}$ crosses $\beta_{t}$. In particular, it is not necessarily the case that $V_{t} \leq \beta_{t}$ for all $t \leq m$. Instead, $m$ is simply the last time when $V_{t}$ is at or below $\beta_{t}$, or else 0 if this never happens. As will be clear, $V_{t}$ is not required for equilibrium characterization prior to $m$, so its value is irrelevant for $t<m$. Finally, note that nothing prevents $m$ from being the final trading opportunity $T$.
} 
offer, $S_{\emptyset}$ can do no better. Only $S_{L}$ could possibly gain in the continuation following a nonequilibrium offer: the Intuitive Criterion requires $B_{\emptyset}$ to conclude that any non-equilibrium offer came from $S_{L}$, and so reject any positive price, yielding zero payoffs.

In periods before $m$ the comparison between $V_{t}$ and $\beta_{t}$ is no longer relevant because $S$ is not expected to skim using price 1 in all subsequent periods. In fact, $S$ is expected to settle in $m$ at the latest. If settlement in the absence of disclosure is anticipated after some time period $t$ then $B$ 's continuation value from rejecting the offer at $t$ is positive, which lowers the maximal settlement price that she would be prepared to accept at $t$.

Indeed, suppose that in period $t$ it is anticipated that $S$ will next settle, along the equilibrium path, in period $k>t$, at price $q_{k}$, but that he will skim for all $\tau \in(t, k) \cap \mathbb{T}$. Then the highest price $B_{H}$ would accept is exactly the skimming price at time $t$, given by

$$
p_{t}=1-\left(\frac{1-r_{S} \Delta}{1+\rho \Delta}\right)^{(k-t) / \Delta}\left(1-q_{k}\right) \text {. }
$$

To see why, note that $B_{H}$ expects a surplus of $1-q_{k}$ at $k$ in precisely $(k-t) / \Delta$ periods, unless $S$ also discovers that the state is high, in which case she gets nothing. $B_{H}$ does not expect any additional surplus before $k$ as she will be skimmed in every period.

Anticipating the next settlement at $k>t$, the highest price that $B_{\emptyset}$ would be prepared to accept is the settlement price at $t$. By similar reasoning, this is

$$
q_{t}=\beta_{t}-\left(\frac{1-r_{S} \Delta}{1+\rho \Delta}\right)^{(k-t) / \Delta} \beta_{t}\left(1-q_{k}\right)+\left(\frac{1-r_{B} \Delta}{1+\rho \Delta}\right)^{(k-t) / \Delta}\left(1-\beta_{t}\right) q_{k} .
$$

Again, the intuition is that $B_{\emptyset}$ has to be compensated for the continuation value she could realize by refusing to buy the good of expected value $\beta_{t}$ at $t$. This surplus is $1-q_{k}$ in period $k$ if the state is high (i.e., with probability $\beta_{t}$ ) provided $S$ does not discover the state by then, less $q_{k}$ if the state is low (i.e., with probability $1-\beta_{t}$ ) provided $B$ herself does not discover the state by then. It is easy to see that $q_{t}<p_{t}$ when the next settlement is anticipated at price $q_{k}$ in period $k>t$. That is, the skimming price targeting $B_{H}$ only is indeed greater than the settlement price targeting both types of $B$.

With the skimming and settlement prices in hand it is straightforward to write down the uninformed seller's expected payoff from skimming versus settling at $t$ when, in equilibrium, the next settlement is anticipated in period $k>t$ in the absence of disclosure. The comparison of these two payoffs determines whether $S$ skims or settles at $t$. Working backwards, the seller's optimal decision can be derived at $t-\Delta$, and so on, which yields the unique equilibrium satisfying the properties described in Section 2.

With the details of the rest of the derivation relegated to the appendix, the following key proposition characterizes the desired equilibrium of the model.

Proposition 1 (Equilibrium Characterization). There exists a unique equilibrium such that

(i) $S$ conceals $s_{t}=L$ and discloses $s_{t}=H$, while $B$ conceals $b_{t}=H$ and discloses $b_{t}=L$; trade occurs immediately after hard evidence disclosure at a price matching $B$ 's valuation;

(ii) B's out-of-equilibrium beliefs satisfy the Intuitive Criterion after $S^{\prime}$ 's deviation; whereas $S_{\emptyset}$ believes $B$ is uninformed if she rejects a price she was supposed to have accepted for sure. 
For all $t \in \mathbb{T}$, absent prior disclosure the seller either skims by setting a price $p_{t}$ accepted by $B$ if and only if $b_{\tau}=H$ for some $\tau \leq t$, or settles by setting a price $q_{t}$ accepted by $B$ after any history.

Define $m=0$ if $V_{t}>\beta_{t}$ for all $t \in \mathbb{T}$, otherwise let $m=\max \left\{t \in \mathbb{T} \mid V_{t} \leq \beta_{t}\right\}$. In the absence of prior disclosure by either player, $S$ skims with price $p_{t}=1$ at any $t>m$. If $m \in \mathbb{T}$ then $S$ settles with price $q_{m}=\beta_{m}$ at $t=m$. For $\rho$ sufficiently small the seller skims in all periods $t<m$.

For $\rho$ small enough, the equilibrium has the wait-settle-wait structure described in the introduction. To provide an intuition for this result, first consider an uninformed seller's decision before some future settlement period $m$. If the players are sufficiently patient, the seller skims, hoping to sell early to an informed buyer concealing $\omega=H$. The seller also gains from all of the chances before $m$ is reached to discover $\omega=H$ for himself. The condition that players be sufficiently patient is natural: in the periods directly before $m$, the seller is willing to skim rather than settle immediately only if the probability that either he or the buyer discovers $\omega=H$ in the intervening time (an upside risk) outweighs his impatience for waiting until $m$. There is also a downside risk that, in between now and $m$, the buyer observes $\omega=L$. Naturally, this risk is reflected in both the seller's continuation value of waiting till $m$ and the highest price the buyer is willing to pay right away.

Now consider a seller who faces the prospect of making a sale only when $b_{t}=H$ or $s_{t}=H$ at some point in the future, but otherwise is unable to sell the good. Given the finite horizon, there is a diminishing number of opportunities left in which to find such evidence and disclose it. At the same time, the buyer is becoming more and more pessimistic. These two forces can be sufficient to drive the seller to offer a price that is immediately acceptable to the buyer regardless of whether or not she is concealing evidence that $\omega=H$. The seller becomes "desperate" enough to settle at some $m \leq T$. Before $m$, however, the seller is no longer worried about running out of time; the buyer is still becoming more pessimistic, but this does not matter since the seller knows a sale will take place for sure at $m$. Moreover, and as mentioned above, there is value in the remaining opportunities to find hard evidence before $m$ is reached: trade takes place at a high price following either disclosure by $S$ or by skimming an informed $B$. It is in a sufficiently patient seller's interest to wait.

Arbitrarily Frequent Interactions. In the following two sections equilibrium play and the corresponding quantities of interest will be further analysed in two, leading cases of interest. First, under a finite time horizon with sufficiently patient players either $S$ will skim to the end or until a unique, final moment of settlement. Second, with an arbitrarily long time horizon it transpires that $S$ will skim in all periods if $\rho=0$, but settle right away for any $\rho>0$ if the period length $\Delta$ is sufficiently short. In both cases the equilibrium pricing paths and comparative statics results are derived and illustrated.

These cases will be analysed under the assumption of frequent interaction. The model where $\Delta>0$ is arbitrarily small is not only theoretically attractive, as a valid approximation of continuous time, but also results in formulæ that make the calculations more palatable. In practical terms, formulæ for price paths and various constraints will be evaluated in the limit as $\Delta \rightarrow 0$. It should be noted that the model is still a properly specified, discrete-time game, with the equilibrium characterized by Proposition 1 for any $\Delta>0$. 
By convention, subscript $t$ will be used to refer to the value of a variable in period $t$ of the discrete-time model with a given $\Delta>0$; however, a variable written as a function with argument $t$ will denote the limit of the variable's value at $t$ as $\Delta \rightarrow 0$. For example, ${ }^{13}$

$$
\beta(t) \equiv \lim _{\Delta \rightarrow 0} \beta_{t}=\frac{\sigma}{\sigma+(1-\sigma) e^{r_{S} t}} .
$$

In the appendix it is shown that, as $\Delta \rightarrow 0, V_{t}$ as defined by (2) and (3) converges to

$$
V(t)=\left[1-e^{-\left(r_{B}+r_{S}+\rho\right)(\bar{t}-t)}\right] \sigma \lambda+e^{-\left(r_{B}+r_{S}+\rho\right)(\bar{t}-t)} \sigma v,
$$

where $\lambda=\left(r_{B}+r_{S}\right) /\left(r_{B}+r_{S}+\rho\right) \in(0,1] . V(t)$ is decreasing and concave for $\rho$ sufficiently small. Note that if $\bar{t}=\infty$, so that the time horizon is unbounded, then the expected payoff to $S_{\emptyset}$ from skimming forever becomes constant in $t$ : (8) becomes $V(t)=\sigma \lambda$.

Recall the definition of $m$, which, when positive, is given by (4). Define $m^{*}$ to be the value of $m$ as $\Delta \rightarrow 0$. To provide a condition for $m^{*}$ to be interior, so that a last moment of settlement occurs strictly within the trading window $(\underline{t}, \bar{t})$, first note that $V(0)<\sigma$ from (8). ${ }^{14}$ Similarly, from (7), $\beta(0)=\sigma$. The buyer's belief is above the seller's continuation value from skimming forever at time 0 . Therefore, if $\underline{t}$ is sufficiently close to zero and the seller's continuation value lies above the buyer's belief at $\bar{t}$, so that $V(\bar{t})>\beta(\bar{t})$, then $m^{*} \in(\underline{t}, \bar{t})$. Now $V(\bar{t})=\sigma v$, so this latter condition is equivalent to

$$
\bar{t}>\frac{1}{r_{S}} \ln \left(\frac{1-\sigma v}{(1-\sigma) v}\right) .
$$

Hence $m^{*} \in(\underline{t}, \bar{t})$ exists if (9) holds and $\underline{t}$ is sufficiently close to 0 . This is necessary and sufficient: if $V(\cdot)$ were to be below $\beta(\cdot)$ both evaluated at $\bar{t}$, then $m^{*}=\bar{t}$ by definition.

Thus, for a wide enough trading window, an interior final moment of settlement exists. Suppose it does. Then, by evaluating (7) and (8) at $t=m^{*}$, equating $\beta\left(m^{*}\right)=V\left(m^{*}\right)$, and rearranging in terms of $m^{*}$, it is straightforward to show that the final moment of settlement converges as $\Delta \rightarrow 0$ to the largest feasible solution of the equation

$$
m^{*}=\bar{t}-\frac{1}{r_{B}+r_{S}+\rho} \ln \left(\frac{\lambda-v}{\lambda-\beta\left(m^{*}\right) / \sigma}\right) .
$$

This expression allows comparative static exercises to be performed: see the next sections. Turning to prices, recall from Proposition 1 that $S$ skims at all $t<m$ if $\rho$ is sufficiently small. The $\Delta \rightarrow 0$ limit of the pre-settlement skimming price in (5) for $k=m^{*}$ is

$$
p(t)=1-\left[1-\beta\left(m^{*}\right)\right] e^{-\left(r_{S}+\rho\right)\left(m^{*}-t\right)} .
$$

Note that $p(t)$ is decreasing in $t$. The skimming price charged by an uninformed seller falls over time because the informed buyer at whom the price is targeted becomes more and more willing to wait till $m^{*}$ and take advantage of the settlement price she will face then. The buyer knows the seller has fewer and fewer opportunities to discover $\omega=H$. Therefore, the seller has to offer a larger and larger discount in his skimming price in order

\footnotetext{
${ }^{13}$ This and all the formulæ for $\Delta \rightarrow 0$ below are derived in the appendix.

${ }^{14}$ To be precise, $V(0)<\sigma$ so long as $\rho>0$ or $\bar{t}<\infty$. The case of $\rho=0$ and $\bar{t}=\infty$ is explored in Section 5 .
} 
to ensure acceptance by the buyer. Naturally, the price the informed buyer is willing to pay must converge to $\beta\left(m^{*}\right)$ at $t=m^{*}$, which is confirmed by inspection of (11).

The next section presents comparative-statics results and explores the consequences for total surplus, welfare, and efficiency in the leading case of interest, when the equilibrium has the skim-settle-skim structure emphasized throughout. It does so by focusing on arbitrarily frequent interactions for algebraic convenience. Nonetheless, many of the results (appropriately restated) can be derived in the discrete-time model with $\Delta \gg 0$ as well.

\section{WAiting to SetTLE}

If the time horizon is sufficiently long then a final moment of settlement $m^{*}$ exists. Moreover, by Proposition 1, if the players are sufficiently patient so that $\rho \leq \bar{\rho}$ for some $\bar{\rho}>0$, then $S$ skims in all trading periods before $m^{*}$. In equilibrium, absent disclosure or $B$ accepting a skimming price, the players wait to settle for a specific amount of time, $m^{*}$.

Figure 1 illustrates this case with a numerical example. The uninformed buyer's belief is drawn in red; the uninformed seller's continuation value from skimming until $\bar{t}$ in blue. ${ }^{15}$ $m^{*}$ is the last moment where the former is above the latter. Thus, the seller skims (out of equilibrium) from $m^{*}$ to $\bar{t}$. At $m^{*}$, the settlement price is $\beta\left(m^{*}\right)$; the pre-settlement skimming price given by $p(t)$ and calculated from (11) is drawn in black.

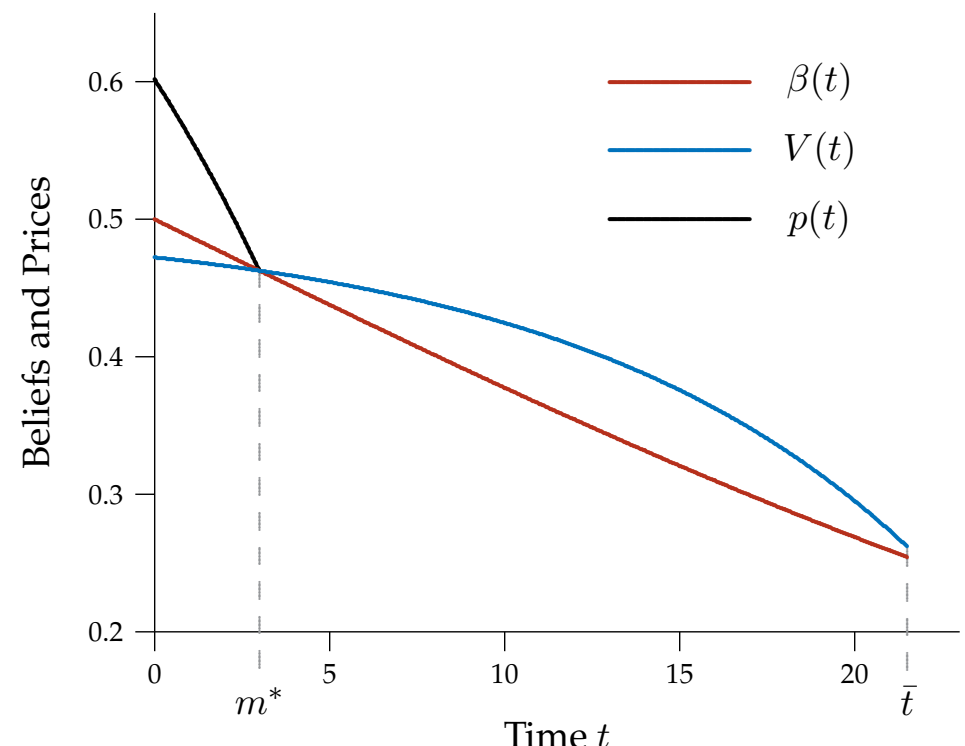

FIGURE 1. Waiting to Settle with a Finite Horizon.

The figure illustrates a case of waiting to settle with a finite horizon $\bar{t}=22$. The parameter values are $r_{B}=r_{S}=0.05, v=\sigma=0.5$, and $\rho=0$. The period length $\Delta>0$ is taken to be arbitrarily short. With these data $\beta(t)<V(t)$ for all $t>m^{*} \approx 3$. So, absent disclosure, $S$ skims with price $p(t)$ at all $t<m^{*}$, settles at $m^{*}$ with price $\beta\left(m^{*}\right) \approx 0.46$, and skims at all $t>m^{*}$ with price $p(t)=1$, off the equilibrium path.

The next result establishes the comparative statics of $m^{*}, \beta\left(m^{*}\right)$ and $p(t)$ for all $t<m^{*}$ in the various parameters of the model. Formal proofs are given in the appendix.

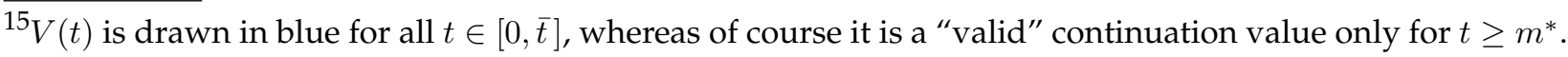


Proposition 2 (Finite Horizon: Comparative Statics). Assume that $\bar{t}<\infty$ is sufficiently high, as in (9), that $\underline{t} \geq 0$ is sufficiently close to zero, and that $\rho \geq 0$ is sufficiently low. Consider arbitrarily frequent interactions with $\Delta \rightarrow 0$. A final moment of settlement exists, and

(i) the $\Delta \rightarrow 0$ limit of the last moment of settlement, $m^{*}$, is increasing in the prior $\sigma$ and discount rate $\rho$, but decreasing in the seller's outside option $v$, the time horizon $\bar{t}$, and the probabilities $r_{B}$ and $r_{S}$ that the players obtain hard evidence;

(ii) the $\Delta \rightarrow 0$ limit of the settlement price, $\beta\left(m^{*}\right)$, is increasing in $v, \bar{t}, r_{B}$ and $r_{S}$, and decreasing in $\rho$. In contrast, the skimming price before settlement, $p(t)$ from (11), uniformly decreases at all $t<m^{*}$ for an increase in $v, \bar{t}$, or $r_{B}$, or for a decrease in $\rho$.

Some of these comparative statics effects can be established simply by "shifting curves"; in particular, by shifting the graph of $V$ in Figure 1. Hence, these are valid no matter how large is $\Delta$. For example, an increase in $\bar{t}$ shifts the graph of $V$ to the right horizontally, without changing its shape, as $V(\bar{t}) \equiv \sigma v$ and $V(t)$ depends on the difference $(\bar{t}-t)$ rather than $t$ itself. The graph of $\beta$ is unchanged for a change in $\bar{t}$, so the last moment of settlement $m^{*}$ where $V$ and $\beta$ intersect must decrease. The settlement price goes up along the $\beta$ curve as $m^{*}$ falls; however, the skimming price at every $t<m^{*}$ decreases, because $B^{\prime}$ s continuation value from rejecting this price goes up. An increase in $v$ or a decrease in $\rho$ also shift the graph of $V$ up and out without affecting $\beta$, hence the equilibrium value of $m^{*}$ goes down, $\beta\left(m^{*}\right)$ goes up, and $p(t)$ for $t<m^{*}$ goes down everywhere.

The results that (i) a longer time horizon $\bar{t}$, so that there is more time to "haggle", or (ii) a lower discount rate $\rho$, so that the players are more patient, or (iii) an increase in the seller's valuation for the good in the high state, $v$, all bring the time of settlement earlier thereby raising the settlement price may seem counterintuitive. However, they all follow from the intuition that at the time of settlement $S$ must be compensated for an increase in his expected payoff from skimming till the end. The only way to achieve this is by settling earlier, before the uninformed buyer becomes too pessimistic.

The fact that the time of agreement moves forward when $r_{B}$ increases is equally straightforward: when the buyer becomes better able to identify the value of the good, the seller is unwilling to wait so long for settlement since his continuation value $V(t)$ is raised, while the buyer's belief $\beta(t)$ is unaffected. The buyer faces lower skimming prices $p(t)$ before settlement: a buyer who is better able to identify $\omega$ can drive down the price she faces. However, the settlement price agreed upon will be higher as settlement takes place earlier when the buyer is still relatively optimistic about the good's value.

Likewise, an increase in $r_{S}$ brings forward the date of settlement and raises the associated price. Now $\beta(t)$ is affected as well as $V(t)$ : the buyer's belief deteriorates faster because nondisclosure by the seller is more indicative of bad news. In order to charge a high price an uninformed seller needs to act relatively quickly. The fact that the seller's continuation value is also raised by an increase in his ability to identify $\omega$ reinforces this effect.

Finally, and more subtly, an increase in the prior $\sigma$ raises $m^{*}$. Although it certainly raises $V(t)$, as a quick inspection of (8) verifies, raising $\sigma$ also flattens and raises $\beta(t)$. In particular, the buyer, being more sure of $\omega=H$, is relatively prepared to ignore silence from 
$S$ and wait. The seller can therefore wait longer even though the rise in his continuation value entails a relatively high price to compensate for skimming after $m^{*}$. While $V(t)$ rises one-to-one with $\sigma$, and in parallel $\beta(t)$ rises with $\sigma$, the flattening of the buyer's beliefs comes in addition, thereby lengthening the time to agreement. Uninformed players who are more optimistic about the value of the good are prepared to spend longer waiting before reaching agreement, a fact which has implications for the induced level of inefficiency: a theme which is the focus of the remainder of this section.

The next proposition concerns total surplus, or social welfare. If a settlement date exists and $S$ skims leading up to it then the delay from skimming when the good is valuable is the only the source of efficiency loss, provided $\rho>0$. At the start of period $t<m$ the expected total surplus conditional on $\omega=H$ but neither player knowing this fact is

$$
w_{t}=\left[r_{S} \Delta+\left(1-r_{S} \Delta\right) r_{B} \Delta\right]+\left(1-r_{S} \Delta\right)\left(1-r_{B} \Delta\right) \frac{w_{t+\Delta}}{1+\rho \Delta},
$$

where the first bracketed expression is the probability that either player discovers $\omega=$ $H$ at the beginning of period $t$ and so $B$ buys right away, whereas the second term is the continuation value of the total surplus in case both players remain uninformed. This difference equation and the boundary condition $w_{m}=1$, from settling at $m$, determine $w_{t}$, the expected total surplus at $t$ conditional on $\omega=H$ not being known to either player.

For arbitrarily frequent interactions, taking the limit as $\Delta \rightarrow 0$, the expected total surplus at $t$ conditional on $\omega=H$ not being known to either player converges to

$$
w(t)=\lambda+(1-\lambda) e^{-\left(r_{B}+r_{S}+\rho\right)\left(m^{*}-t\right)},
$$

with $\lambda=\left(r_{B}+r_{S}\right) /\left(r_{B}+r_{S}+\rho\right)$, as before. This can be verified easily by first computing

$$
\dot{w}(t)=\lim _{\Delta \rightarrow 0} \frac{w_{t+\Delta}-w_{t}}{\Delta}=-\left(r_{B}+r_{S}\right)+\left(r_{B}+r_{S}+\rho\right) w(t)
$$

from (12) and integrating it up with the boundary condition $w\left(m^{*}\right)=1$.

The next proposition concerns the comparative statics of ex-ante expected social surplus, denoted $W$, in the various parameters. Define this measure of welfare as $W \equiv \sigma w(0)$, so

$$
W=\sigma\left[\lambda+(1-\lambda) e^{-\left(r_{B}+r_{S}+\rho\right) m^{*}}\right] .
$$

Proposition 3 (Finite Horizon: Welfare). Consider arbitrarily frequent interactions with $\Delta \rightarrow 0$. Let $\bar{t}<\infty$ be sufficiently high, as in (9), $\underline{2} \geq 0$ be sufficiently close to zero, and $\rho \geq 0$ be sufficiently low so that a final moment of settlement $m^{*}$ exists. $W$ is increasing in the probabilities $r_{B}$ and $r_{S}$ that the players obtain hard evidence, decreasing in the discount rate $\rho$, and increasing in the seller's salvage value $v$ and time horizon $\bar{t}$. Increasing the prior $\sigma$ can reduce $W$.

Greater probabilities that the players obtain hard evidence bring the settlement date forward as well as increase the probability of trade from discovering $\omega=H$; total surplus increases on both accounts. Decreasing $\rho$ brings the settlement date forward and decreases the cost of delay, too. Increases in $v$ and $\bar{t}$ affect $m^{*}$ only, reducing delay, hence both are socially beneficial. However, a higher $\sigma$ leads to a longer delay, for reasons discussed above, which may outweigh the increase in the probability that the good is valuable. In this sense, bargaining over a good of higher value may result in lower surplus. 
The next section turns to the case of $\bar{t}=\infty$. While the preferred model is the finite horizon case, since this admits the possibility that the good's true value is never discovered, the unbounded horizon case is of interest for technical reasons, as a natural limit of the former case, as well as being commonly assumed in many bargaining models in the literature.

\section{INFINITE HORIZON}

This section analyses the limit of equilibrium behaviour as the time horizon $\bar{t}$ converges to infinity. Limiting equilibrium behaviour is sensitive to certain parameter values: in particular, the equilibrium under an infinite horizon exhibits immediate settlement for any $\rho>0$ provided $\underline{t}=0$ and $\Delta>0$ is arbitrarily small; in contrast, the seller skims forever if $\rho=0$. Of course, these features can be understood as rather natural limits of the equilibrium structure already described earlier in Proposition 1.

For a fixed period length $\Delta>0$, the uninformed seller's expected payoff from skimming from $t$ onwards will be denoted by $\tilde{V}_{t}$; the tilde decoration refers to the infinite horizon case throughout. $\tilde{V}_{t}$ has to be constant in $t$ : at any two times $t$ and $t^{\prime}$ following histories in which all the price offers were rejected by $B$ and nothing was disclosed, the future must look the same to $S_{\emptyset}$. That is, $\tilde{V}_{t}$ is the solution to (3) with $\tilde{V}_{t}=V_{t} \equiv V_{t+\Delta}$. Therefore

$$
\tilde{V}_{t}=\frac{r_{B}+r_{S}+\left(\rho-r_{S}\right) r_{B} \Delta}{r_{B}+r_{S}+\rho-r_{B} r_{S} \Delta} \hat{\sigma}
$$

where $\hat{\sigma}=\sigma /\left[1-(1-\sigma) r_{B} \Delta\right]>\sigma$ is the uninformed seller's belief that $\omega=H$. Recall this exceeds the prior because $B$ might be concealing a just-received $H$ signal.

A first observation is that when $\bar{t}$ grows unboundedly, with frequent interaction and sufficiently patient players, an interior final moment of settlement fails to exist: in equilibrium $S$ skims forever. ${ }^{16}$ To see this, note that for $\rho=0$ the seller's payoff from skimming forever becomes $\tilde{V}_{t}=\hat{\sigma}$, which strictly exceeds $\beta_{t}$ for all $t \in\{\Delta, 2 \Delta, \ldots\}$ even if $\Delta>0$ is arbitrarily small. Hence $\tilde{V}_{t}>\beta_{t}$ for all $t$, that is, $S$ skims forever when the horizon is infinite and $\rho$ is sufficiently close to zero. In particular, the conclusion that $S$ skims forever applies for any $\Delta>0$ and with no discounting, $\rho=0$. This result has much in common with "no-trade theorems" familiar from the literature following Milgrom and Stokey (1982).

However, it is also true that for any given $\rho>0$, there exists a sufficiently small period length $\Delta>0$ that induces $S$ to settle in finite physical time. To see this note that

$$
\lim _{\Delta \rightarrow 0} \tilde{V}_{t}=\frac{\left(r_{B}+r_{S}\right) \sigma}{r_{B}+r_{S}+\rho} \equiv \sigma \lambda<\sigma=\lim _{t \rightarrow 0} \beta(t) .
$$

Therefore, for any given $\rho>0$, the seller won't skim forever provided $\Delta>0$ is small enough, because $\tilde{V}_{t}>\beta_{t}$ fails for $t=\Delta>0$ sufficiently close to zero.

Now fix $\rho>0$ and assume $\Delta>0$ is sufficiently small so that there indeed exists an interior final moment of settlement, $m>0$. Both the time and the price of settlement depend on $\Delta$;

\footnotetext{
${ }^{16}$ In contrast, recall that $m^{*}$ solving (10) exists when $\bar{t}$ is finite but sufficiently large.
} 
for a fixed $\rho>0$, as $\Delta \rightarrow 0$, these quantities converge to

$$
\tilde{m}=\frac{1}{r_{S}} \ln \left(\frac{\rho+(1-\sigma)\left(r_{B}+r_{S}\right)}{(1-\sigma)\left(r_{B}+r_{S}\right)}\right) \quad \text { and } \quad q(\tilde{m})=\frac{\left(r_{B}+r_{S}\right) \sigma}{r_{B}+r_{S}+\rho} \equiv \sigma \lambda .
$$

At any $t<\tilde{m}$ the seller may skim or settle at prices strictly less than 1 or $\beta_{t}$ respectively, because $B$ anticipates settling at $\tilde{m}$. The perhaps surprising fact demonstrated in the following proposition is that for any given $\rho>0$, if $\Delta>0$ is arbitrarily small then $S$ settles right away when the parties meet for the first time at $\Delta$. Moreover, $S$ offers a settlement price even off the equilibrium path in all time periods $t<\tilde{m}$.

Proposition 4 (Infinite Horizon: Equilibrium Settlement). Let $\underline{t}=0$ and $\bar{t}=\infty$.

(i) For any fixed $\Delta>0$, if $\rho \geq 0$ is sufficiently small then in equilibrium $S$ skims in every period with a price of 1 , which $B$ accepts iff either she has discovered or $S$ has proved $\omega=H$. If $\rho=0$ then, for any $\Delta>0, S$ skims forever with price $p(t) \equiv 1$.

(ii) For any fixed $\rho>0$, if $\Delta>0$ is sufficiently small then the equilibrium involves a last moment of settlement $m>0$, moreover, $S$ immediately settles at $t=\Delta$ and does so even off the equilibrium path for all $t \leq m$, but skims forever for all $t>m$.

(iii) For any fixed $\rho>0$, as $\Delta \rightarrow 0$, the last moment of settlement and the final settlement price converge to the quantities given in (14). The settlement price for $t \leq \tilde{m}$ converges to $q(t)$ such that $q(\tilde{m})=\beta(\tilde{m})=\sigma \lambda, q(t)<\beta(t)$ for $t<\tilde{m}$, and for all $t \in[0, \tilde{m}]$,

$$
\dot{q}(t)=\left(\rho+r_{B}\right)[1-\beta(t)] q(t)-\left(\rho+r_{S}\right) \beta(t)[1-q(t)] .
$$

The case of skimming forever is a natural limit of the result described in the last section: Proposition 2 states that the last moment of settlement, $m^{*}$, is decreasing in the time horizon $\bar{t}$. As the horizon grows unboundedly, $m^{*}$ shrinks towards zero, and eventually is smaller than $\Delta$ if $\underline{t}=0$. Effectively, the trading parties have missed their opportunity to reach agreement by the time they first meet, and the seller is sufficiently patient to wait for hard evidence to be discovered by either party and then sell at the maximal price.

The proof of the surprising bit, that for any $\rho>0$ settlement occurs at all $t \in\{\Delta, \ldots, m\}$ provided $\Delta$ is sufficiently small, is given in the appendix. It is based on an induction argument: if $S_{\emptyset}$ anticipates that settling will be worthwhile a short time later at a price that $B_{\emptyset}$ will find acceptable, knowing that settlement will be offered in every period up until and including $m$, then it is optimal for $S_{\emptyset}$ to settle in the current period as well.

The players' impatience is crucial. Recall that the gap between the seller's and buyer's pre-settlement beliefs makes it possible for the seller to delay before $m$. Moreover, the settlement price offered at $m$ is relatively low as, after $m$ and off the equilibrium path, $S$ fears running out of time to discover $\omega$ and is prepared to discount the price heavily to make a sale to an increasingly pessimistic buyer. When $S$ has an infinite horizon after $m$, he has no such fear and can offer a relatively high and early settlement price. Knowing this, before settlement, the incentive for $S$ to wait till $m$ is reduced, and the importance of the gap between beliefs is eroded by impatience. As the length of the time period $\Delta$ shrinks, the chance either player receives a signal between times $m-\Delta$ and $m$ shrinks with it. Eventually, for $\rho>0$, impatience outweighs this chance, and the trading parties 
can find a mutually agreeable settlement price at $m-\Delta$ as well. Clearly this argument does not particularly depend upon $m$, and so by induction, the parties settle at all $t<m$.

There is no contradiction between the findings that

(i) for any fixed $\Delta>0, S$ strictly prefers to skim in all periods before $m$ provided $\rho \geq 0$ is sufficiently small, which is true no matter whether the time horizon is finite or infinite (Proposition 1); and

(ii) for any fixed $\rho>0, S$ settles in all periods at and before $m$, provided $\Delta>0$ is sufficiently small, which is true under an infinite horizon (Proposition 4).

First, the order of quantifiers is different in these two statements. Second, with $\bar{t} \rightarrow \infty$ and $\rho=0$ there is no interior final moment of settlement: $S$ skims forever. Hence there is only an apparent discontinuity of the latter settling-at-or-before- $m$ result as $\rho$ approaches 0 .

The derivation of $\tilde{m}$ for a particular numerical example is illustrated in Figure 2, an infinitehorizon version of Figure 1. The uninformed seller's expected payoff from skimming forever is constant in time, converging to $\sigma \lambda$ as $\Delta \rightarrow 0$. The horizontal graph of $\tilde{V}$, in blue, intersects the graph of $\beta$, in red, exactly once, at $t=\tilde{m}$. The settlement price, $q(t)$ for $t \leq \tilde{m}$, is drawn in black. Settlement takes place immediately at price $q(\underline{t})$.

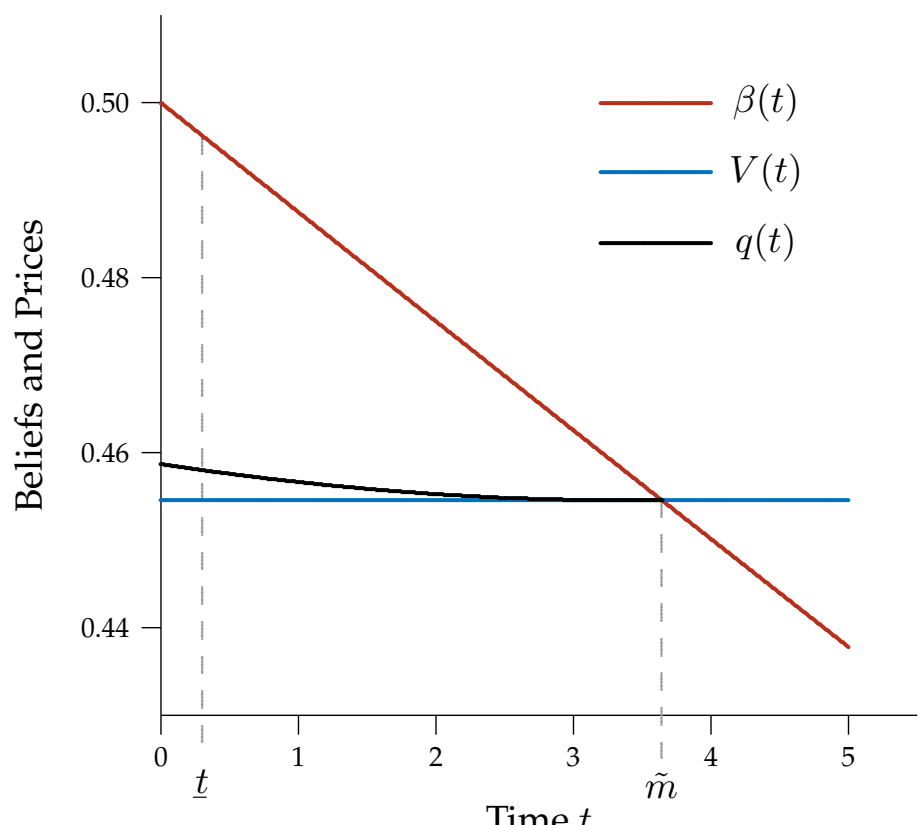

FIGURE 2. Immediate Settlement with an Infinite Horizon.

The figure illustrates a case of immediate settlement with an infinite horizon. The parameter values are $\sigma=0.5, r_{B}=r_{S}=0.05$, and $\rho=0.01$. The uninformed seller's continuation value is constant in time; $\tilde{V} \rightarrow \sigma \lambda \approx 0.455$ as $\Delta \rightarrow 0$. $\beta(t)$ intersects $\tilde{V}$ exactly once at $\tilde{m} \approx 3.65$. Thus $q(\tilde{m})=\beta(\tilde{m}) \approx 0.455$. Settlement takes place immediately at the price given by $q(\underline{t})$ and, off equilibrium path, a settlement price of $q(t)$ is offered at all subsequent $t \leq \tilde{m}$. After $\tilde{m}, S$ skims with price 1 .

This section concludes with comparative statics results regarding the final settlement date and the price at which immediate settlement occurs, with a fixed $\rho>0$ and arbitrarily 
small $\Delta>0$, in the infinite-horizon case. A greater common optimism about the good's value, that is a higher prior $\sigma$, or more patience, a lower discount rate $\rho$, increase the price at which the parties settle at the first trading opportunity.

Proposition 5 (Infinite horizon: Comparative Statics). Let $\underline{t}=0, \bar{t}=\infty$, fix $\rho>0$, and let $\Delta>0$ be arbitrarily small. The $\Delta \rightarrow 0$ limit of the final moment of settlement, $\tilde{m}$, is increasing in $\sigma$ and $\rho$ and decreasing in $r_{B}$ and $r_{S}$. If $r_{B}=r_{S}=r$ then the price at which immediate settlement occurs, $q(\underline{t})$, is increasing in $\sigma$ and increasing in patience for $\rho$ sufficiently low.

The comparative statics of $\tilde{m}$ follow straightforwardly from an inspection of (14). The proofs of the results on the immediate settlement price, $q(\underline{t})$, are more subtle and are relegated to the appendix. For example, an increase in $\sigma$ shifts both curves in Figure 2 up or out, and the new intersection occurs at a higher $\tilde{m}$ and $\beta(\tilde{m}) \equiv \sigma \lambda$; the proof establishes that all settlement prices before $\tilde{m}$ are higher as well.

A final observation is that, since trade occurs immediately with a fixed $\rho>0$ and arbitrarily frequent interaction, there is never any efficiency loss.

\section{RELATED LiterATURE AND CONCLUDING REMARKS}

The main, novel feature of the above model is that the parties may produce and selectively disclose verifiable evidence while participating in a dynamic trading game. As a result, the paper is related to the rich, but mostly distinct literatures on communication games, especially with "hard" information, and dynamic bargaining models.

Information structures with partially or fully verifiable evidence have mainly been applied in information transmission games rather than in bargaining (see Milgrom, 2008, for a survey). There, the central themes are whether communication results in the full revelation of all verifiable information (e.g., Seidmann and Winter, 1997; Mathis, 2008), and whether or not it is beneficial for a player to be known to be more able to generate hard evidence (e.g., Shin, 1994). One of the profound insights in this literature is that verifiability is a two-edged sword: it increases the credibility of the sender, but also the incredulity of the listener in case no message is forthcoming. This effect is clearly at work in the model here, albeit embedded in a model of dynamic price discrimination. Full "unravelling" (first identified in Grossman, 1981; Milgrom, 1981), whereby a silent informed party causes the uninformed party to make a worst-case-scenario inference, does not occur here because it is entirely possible that non-disclosure reflects genuine ignorance. ${ }^{17}$

There is a growing literature on dynamic information-transmission games, both with unverifiable messages (long cheap talk: Aumann and Hart, 2003), and using state-dependent message spaces (long persuasion: Forges and Koessler, 2008). In this line of research the motivation here is perhaps closest to that of Hörner and Skrzypacz (2016). They study a specific persuasion technology with repeated binary-outcome testing in a dynamic game

\footnotetext{
${ }^{17}$ Models in which (one-sided) good information is disclosed, bad concealed, thus making the uninformed party relatively pessimistic can be found elsewhere in the literature. For instance, this logic is at work in Shin (2003), albeit in a very different model of a firm manager disclosing information to a financial market.
} 
where communication and certain actions, in their case payments, are concurrent. ${ }^{18}$ The sender-optimal equilibrium in their model exhibits a gradual release of information; in contrast, here, essentially by assumption, evidence disclosure immediately ends the game. The dynamic bargaining game in this paper is also very different in structure and purpose from their sender-receiver game.

An early example of a trading model that involves hard information acquisition is that of Shavell (1994), where the focus is on the (in)efficiency of information acquisition rather than on the effects of verifiability itself. Eső and Wallace (2014) compare 'soft' and 'hard' evidence in a much simpler bargaining problem. In a single-period game with two-sided incomplete information, the equilibrium when the seller's information is unverifiable and the equilibrium when his information is verifiable are qualitatively different. Overall the seller is ex-ante better off when his signal is hard. More recently, Koessler and Skreta (2017) study a bilateral asymmetric-information monopoly setting in which the seller is able to provide evidence about the good's characteristics. ${ }^{19}$ The game is static and the focus is on the mechanism design problem faced by the profit-maximizing seller. ${ }^{20}$

The dynamic bargaining game, albeit augmented here with the probabilistic acquisition and voluntary disclosure of hard evidence, is familiar from the literature on dynamic monopoly and the Coase conjecture. ${ }^{21}$ In these dynamic price-discrimination problems, as here, bargaining power is one-sided (the seller makes all the take-it-or-leave-it offers) and a central question is what happens as the period-length, representing the seller's ability to commit to a price, converges to zero. Recent papers by Fuchs and Skrzypacz $(2010,2013)$ study the stochastic arrival of new traders and deadlines in this type of model, whereas Daley and Green $(2012,2016)$ analyse the effects of the gradual, exogenous public revelation of one side's private information. In contrast to all of these analyses, here both parties may acquire private information over time and evidence is verifiable-concealable: it is neither "soft" private information nor public "news". Despite the differences in modelling information structures, there are some shared conclusions, for instance, concerning the possibility of delay on the equilibrium path. ${ }^{22}$ Comparative statics results are difficult to compare because of the differences in modelling assumptions, but a common relationship

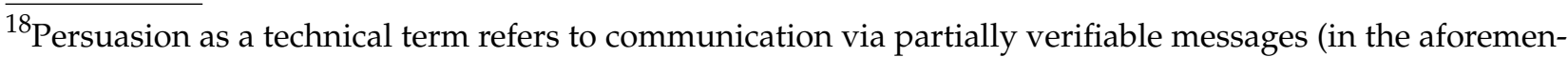
tioned Shin, 1994, for instance) and, relatedly, to the contractible design of a decision-maker's information structure (in the Bayesian persuasion literature, see Kamenica and Gentzkow, 2011). The assumptions in this literature are very different from those made here, however. In the current model, both parties may be able to affect what the other knows over time by way of verifiable disclosure, thereby persuading the other of the good's value. Evidence arrives stochastically and may only be either disclosed or concealed. Hence the game here is less related to persuasion problems of the latter type.

${ }^{19}$ On the other hand, and again with one-sided asymmetric information, Sher and Vohra (2015) study the situation in which it is the buyer who can disclose valuation-relevant evidence to the monopolist.

${ }^{20} \mathrm{~A}$ motivation for this latter paper is provided by the quality-disclosure and certification literature. Again see Dranove and Jin (2010), as well as the more recent contributions from Koessler and Renault (2012), in a price-posting world, and Koessler and Skreta (2016), when the seller offers more general mechanisms.

${ }^{21}$ This literature has a long history, seminal papers include Stokey (1981), Bulow (1982), Fudenberg, Levine, and Tirole (1985), Gul, Sonnenschein, and Wilson (1986), and Hart and Tirole (1988).

${ }^{22}$ Delay does not necessarily contradict the Coase conjecture. For instance, in the model of Fuchs and Skrzypacz (2010), which exhibits equilibrium delay, the seller's ex-ante profit is the same as if he waited for his outside option to arrive. In contrast, Board and Pycia (2014) show the failure of the Coase conjecture in an environment where the party with an informational advantage has an outside option.
} 
emerges between prices and delay: longer delay, or a longer period of skimming before settlement, is associated with a lower settlement price.

The leading case of Section 4 features a finite horizon, a property it has in common with earlier analyses going back to Ståhl's (1972) doctoral dissertation. Deadline effects including inefficient delay are studied in Sobel and Takahashi (1983), Dekel (1990), Fershtman and Seidmann (1993), and Yildiz (2004) under imperfect or incomplete information about valuations or bargaining strengths. ${ }^{23}$ None of these papers studies evidence disclosure.

Explanations of delay in bargaining also include signalling (Admati and Perry, 1987) or a concern for reputation (Abreu and Gul, 2000). In the former, patient players signal their strength by refusing to agree for a while; in the latter, rational bargainers might strike postures to appear as "irrational types" by making demands and rejecting offers in order to build reputations and get better deals. Another intuitive reason for delay may be excess optimism (for instance, different priors about the distribution of bargaining power, or other behavioural anomalies), although Yildiz (2003) points out the limits of this type of explanation. This literature is related to the extent that delay may be generated on the equilibrium path here as well: the reasons (concealment of hard evidence leading to the impossibility of mutually-acceptable trade) are markedly different, however. Recently, Hwang and Li (2017) derive delay from the privately observed arrival of a bargaining party's outside option. In contrast, here, concealable hard evidence may arise concerning the value of trade within the buyer-seller relationship, that is, as an inside option.

There are many different directions for future research. One, mentioned earlier in Footnote 5 , is to consider imperfect hard evidence production in which there is some probability of receiving the high signal in the low state, and vice versa. A second would be to consider different trading environments, for instance, different surpluses in the two states or a continuum of states. Finally, all bargaining power is held by the seller in this model in order to map more closely to the dynamic-trading literature discussed above. A different approach might model bargaining power shared between the trading parties, for example via alternating offers à la Ståhl (1972) and Rubinstein (1982), or random offers.

\section{APPENDIX. PROOFS AND DERIVATIONS}

Proof of Proposition 1. The existence and uniqueness of an equilibrium satisfying conditions (i) and (ii) were established (by construction) in the text. It remains to show that the seller skims for all $t<m$ provided $\rho>0$ is sufficiently low. This is proved (with strict preference for skimming) for the case of $\rho=0$, then the claim for $\rho$ near zero follows by continuity.

If, at $t<m$, the next time of settlement absent disclosure is $m$ at price $q_{m}=\beta_{m}$, then, by (5) and (6), the skimming price is $p_{t}=1-\left(1-r_{S} \Delta\right)^{(k-t) / \Delta}\left(1-\beta_{m}\right)$, and the settling price can be rewritten

$$
q_{t}=\left[1-\left(1-r_{S} \Delta\right)^{(m-t) / \Delta}\right] \beta_{t}+\left[\left(1-r_{S} \Delta\right)^{(m-t) / \Delta} \beta_{t}+\left(1-r_{B} \Delta\right)^{(m-t) / \Delta}\left(1-\beta_{t}\right)\right] \beta_{m} .
$$

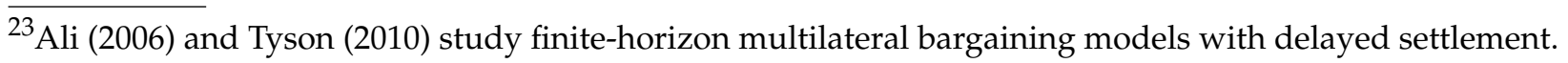


Define $V_{t}^{m}$ as the expected payoff of $S_{\emptyset}$ in period $t$ conditional on no disclosure at or before $t$, from skimming in all periods from $t$ leading up to $m$ (including $t$ but not $m$ ):

$$
V_{t}^{m}=\left[1-\left(1-r_{S} \Delta\right)^{(m-t) / \Delta}\right] \hat{\sigma}+\left[\left(1-r_{S} \Delta\right)^{(m-t) / \Delta} \hat{\sigma}+\left(1-r_{B} \Delta\right)^{(m-t) / \Delta}(1-\hat{\sigma})\right] \beta_{m} .
$$

The seller gets a payoff of 1 if $\omega=H$ (i.e., with probability $\hat{\sigma}$ ) and he finds this out at or before $m$; whereas he gets $\beta_{m}$ provided either the state is $\omega=H$ and he does not discover it or $\omega=L$ and $B$ does not discover it by $m$. By (16) and (17), skimming is more profitable for $S_{\emptyset}$ at $t$ than settling, $V_{t}^{m}>q_{t}$, iff $\hat{\sigma}>\beta_{t}$. The latter, however, always holds by $\hat{\sigma}>\sigma=\beta_{0}>\beta_{t}$ for all $t>0$.

It remains to argue that, as mentioned in Footnote 10, delaying by offering a price exceeding $B^{\prime}$ s willingness to pay is never in the uninformed seller's interests. In fact, $S_{\emptyset}$ is strictly worse off doing so if $r_{B}>0$ and $\rho>0$. Consider $t>m$. The skimming price is 1 , which is accepted by $B$ with positive probability so long as $r_{B}>0$. The best $S$ can get is 1 from disclosing or skimming in future periods, but if $\rho>0$ then $S$ would be strictly better off trying to sell now. At $m$, settling is better than skimming which, by the very same argument, is better than delay.

Now consider $t<m$. Skimming takes place at price (5). This price exactly captures the value that $S$ receives from the possibility of discovering $\omega=H$ in any of the periods up to $m$. Thus, by delaying rather than skimming, $S$ foregoes an opportunity to receive this value at $t$ with some positive probability (since $r_{B}>0$ ) and instead gets at most this value a period later (which is costly, since $\rho>0$ ). Either way, at all $t$, delaying is strictly worse than skimming if $r_{B}, \rho>0$.

Derivation of Formulx for $\Delta \rightarrow 0$. Difference $\beta_{t}$ given in (1) and divide by $\Delta$ to get

$$
\frac{\beta_{t+\Delta}-\beta_{t}}{\Delta}=\frac{-r_{S}\left(1-\beta_{t}\right) \beta_{t}}{\left(1-r_{S} \Delta\right) \beta_{t}+\left(1-\beta_{t}\right)} \text {. }
$$

Take the $\Delta \rightarrow 0$ limit to get $\dot{\beta}(t)=-r_{S} \beta(t)[1-\beta(t)] \in[-1,0]$. Integrating this bounded derivative with boundary condition $\beta(0)=\sigma$ yields $\beta(t)$ as given in (7).

To derive $V(t)$ rearrange (3), cross-divide it by $\Delta$, and take $\Delta \rightarrow 0$ to get

$$
\dot{V}(t) \equiv \lim _{\Delta \rightarrow 0} \frac{V_{t+\Delta}-V_{t}}{\Delta}=\left(r_{S}+r_{B}+\rho\right) V(t)-\left(r_{S}+r_{B}\right) \sigma .
$$

With boundary condition $V(\bar{t})=\sigma v$ the closed-form solution in (8) is obtained.

The formula for $m^{*}$ in the limit as $\Delta \rightarrow 0$, given in (10), follows from rearranging

$$
\beta\left(m^{*}\right)=V\left(m^{*}\right) \equiv\left[1-e^{-\left(r_{B}+r_{S}+\rho\right)\left(\bar{t}-m^{*}\right)}\right] \sigma \lambda+e^{-\left(r_{B}+r_{S}+\rho\right)\left(\bar{t}-m^{*}\right)} \sigma v .
$$

To obtain $p(t)$ in (11) take the limit of $p_{t}$ from (5) as $\Delta \rightarrow 0$ and set $k=m^{*}$.

Proof of Proposition 2. Note that changes in $\bar{t}, v$ and $\rho$ only affect $V$. It is immediate that an increase in $\bar{t}$ or $v$ shifts $V$ out (to the right or up), hence the locus of the last intersection between $\beta$ and $V$ (where both functions are decreasing, with $\beta$ decreasing more rapidly) must decrease. Hence the final moment of settlement decreases and the corresponding settlement price increases (along $\beta$ ) for an increase in $\bar{t}$ or $v$. The skimming price in all periods before settlement decreases because

$$
\frac{\partial p(t)}{\partial m^{*}}=\left[r_{S}+\rho-r_{S} \beta\left(m^{*}\right)\right]\left[1-\beta\left(m^{*}\right)\right] e^{-\left(r_{S}+\rho\right)\left(m^{*}-t\right)}>0
$$

by (11). The opposite is true for an increase in $\rho$. This is because increasing $\rho$ reduces $V$ at all $t<\bar{t}$, but not at $t=\bar{t}$. To see this suppose the reverse: that $V(t)$ remains the same or rises at $t<\bar{t}$ as $\rho$ increases. Then, by (18), the slope of $V$ becomes greater (less negative) at $t$, contradicting the 
requirement that $V$ remains fixed at $t=\bar{t}$. Hence an increase in $\rho$ indeed rotates $V$ counterclockwise (around its terminal value at $\bar{t}$ ), hence $m^{*}$ increases, $\beta\left(m^{*}\right)$ falls, but $p(t)$ goes up for all $t<m^{*}$.

The next claim is that $\partial m^{*} / \partial r_{B}<0$. By inspecting $\dot{V}(t)$ in (18), and noting $V(t)<\sigma$, it is immediate that $\dot{V}(t)$ decreases (becomes more negative) for an increase in $r_{B}$, holding $V(t)$ fixed. Since $V(\bar{t}) \equiv$ $\sigma v$ must remain the same even as $r_{B}$ rises, it follows that $V(t)$ must increase for all $t<\bar{t}$ with an increase in $r_{B}$. Since $\beta$ does not depend on $r_{B}$, it then follows that the equilibrium level of $m^{*}$ decreases. This implies $\beta\left(m^{*}\right)$ increases but $p(t)$ decreases for all $t<m^{*}$.

Now, $\partial m^{*} / \partial r_{S}<0$ since a rise in $r_{S}$ increases $V(t)$ at all $t<\bar{t}$, just as a rise in $r_{B}$ did; moreover, raising $r_{S}$ also causes $\beta(t)$ to fall at all $t>0$. Both effects result in a lower $m^{*}$ and higher $\beta\left(m^{*}\right)$.

Finally, to see $\partial m^{*} / \partial \sigma>0$, first note that $\beta\left(m^{*}\right) / \sigma=\left[\sigma+(1-\sigma) e^{r_{S} m^{*}}\right]^{-1}$ implies

$$
\frac{d}{d \sigma}\left[\frac{\beta\left(m^{*}\right)}{\sigma}\right]=\left(\frac{\beta\left(m^{*}\right)}{\sigma}\right)^{2}\left[e^{r_{S} m^{*}}-1-\frac{\partial m^{*}}{\partial \sigma}(1-\sigma) r_{S} e^{r_{S} m^{*}}\right] .
$$

Hence, by differentiating (10) in $\sigma$,

$$
\begin{aligned}
\frac{\partial m^{*}}{\partial \sigma}=\underbrace{\frac{1}{r_{B}+r_{S}+\rho} \frac{\lambda-\beta\left(m^{*}\right) / \sigma}{\lambda-v}\left(\frac{\beta\left(m^{*}\right)}{\sigma}\right)^{2}}_{K} \times\left[e^{r_{S} m^{*}}-1-\frac{\partial m^{*}}{\partial \sigma}\right. & \left.(1-\sigma) r_{S} e^{r_{S} m^{*}}\right] \\
& =\frac{K\left(e^{r_{S} m^{*}}-1\right)}{1+K(1-\sigma) r_{S} e^{r_{S} m^{*}}}>0,
\end{aligned}
$$

where $K$ denotes the positive factor in front of the bracketed expression in the first equation for $\partial m^{*} / \partial \sigma$ directly above. The overall sign of $\partial m^{*} / \partial \sigma$ follows from $e^{r_{S} m^{*}}>1$.

Proof of Proposition 3. The results follow rather directly from (13).

By the definition of $\lambda$, it is increasing in $r_{B}$ or $r_{S}$. $\lambda$ is the relative weight on $1>e^{-\left(r_{B}+r_{S}+\rho\right) m^{*}}$ in the bracketed term in (13), hence $W$ increases with $r_{B}$ or $r_{S}$, holding $m^{*}$ fixed. However, $\partial m^{*} / \partial r_{i}<0$ for $i=B, S$, therefore $e^{-\left(r_{B}+r_{S}+\rho\right) m^{*}}$ will rise as well, increasing $W$ even further.

Raising $\rho$ reduces $\lambda$ and so $W$, for given $m^{*}$. However, $m^{*}$ is increasing in $\rho$, further reducing $W$.

Both $v$ and $\bar{t}$ reduce $m^{*}$ (leading to an increase in $W$ ) but otherwise do not affect $W$ directly.

Finally, the direct effect of $\sigma$ on $W$ is positive, but the indirect effect through increasing $m^{*}$ is negative, hence the sign of $\partial W / \partial \sigma$ is ambiguous.

Proof of Proposition 4. The claim that for any given $\Delta>0$ and sufficiently small $\rho \geq 0$ the equilibrium prescribes for $S$ to skim in every period was proved in the text.

It was also shown in the text that for any given $\rho>0$ and sufficiently small $\Delta>0$, in equilibrium, there is a last moment of settlement $m>0$ where $\tilde{V}_{m}=\beta_{m}$. Now it will be shown by induction that for all $t<m$ the seller settles given any $\rho>0$, for $\Delta>0$ sufficiently small.

Write the uninformed seller's expected payoff at $t$, conditional on no disclosure at or before $t$, from skimming at $t$ but then settling at price $q_{t+\Delta}$ in $t+\Delta$, as

$$
V_{t}^{t+\Delta}=\hat{\sigma} r_{B} \Delta p_{t}+\hat{\sigma} \frac{\left(1-r_{B} \Delta\right) r_{S} \Delta}{1+\rho \Delta}+\left[\hat{\sigma}\left(1-r_{B} \Delta\right)\left(1-r_{S} \Delta\right)+(1-\hat{\sigma})\left(1-r_{B} \Delta\right)\right] \frac{q_{t+\Delta}}{1+\rho \Delta} .
$$

The first term is $S^{\prime}$ 's payoff from $B_{H}$ accepting $p_{t}=1-\left(1-r_{S} \Delta\right)\left(1-q_{t+\Delta}\right) /(1+\rho \Delta)$ as defined by (5); the second term is his payoff if $S$ himself finds out $\omega=H$ within the current period and charges 1 next time. The third term is $S^{\prime}$ s expected payoff from settling at $t$ : he will get $q_{t+\Delta}$ if either $\omega=H$ but neither player finds it out, or $\omega=L$ and $B$ does not learn it by next time. 
After substituting in $p_{t}$ and rearranging,

$$
V_{t}^{t+\Delta}=\hat{\sigma}\left(r_{B} \Delta+\frac{1-r_{B} \Delta}{1+\rho \Delta}\right)-\frac{1-r_{S} \Delta}{1+\rho \Delta} \hat{\sigma}\left(1-q_{t+\Delta}\right)+\frac{1-r_{B} \Delta}{1+\rho \Delta}(1-\hat{\sigma}) q_{t+\Delta} .
$$

Next, the settlement price at $t$ defined by (6) is

$$
q_{t}=\beta_{t}-\frac{1-r_{S} \Delta}{1+\rho \Delta} \beta_{t}\left(1-q_{t+\Delta}\right)+\frac{1-r_{B} \Delta}{1+\rho \Delta}\left(1-\beta_{t}\right) q_{t+\Delta} .
$$

Uninformed $S$ wants to settle at $t$ whenever $V_{t}^{t+\Delta}<q_{t}$, equivalently, after a cross-multiplication by $(1+\rho \Delta)$, cancelling terms and cross-dividing by $\Delta>0$,

$$
\hat{\sigma} \rho \Delta r_{B}+r_{S} \hat{\sigma}\left(1-q_{t+\Delta}\right)-r_{B}(1-\hat{\sigma}) q_{t+\Delta}<\rho \beta_{t}+r_{S} \beta_{t}\left(1-q_{t+\Delta}\right)-r_{B}\left(1-\beta_{t}\right) q_{t+\Delta} .
$$

After further rearrangement, $V_{t}^{t+\Delta}<q_{t}$ is equivalent to

$$
\left(\hat{\sigma}-\beta_{t}\right)\left[r_{B} q_{t+\Delta}+r_{S}\left(1-q_{t+\Delta}\right)+\rho\right]<\rho \hat{\sigma}\left(1-r_{B} \Delta\right) .
$$

The proof is completed by observing that this condition indeed holds for any $t<m$ provided $\Delta>0$ is sufficiently small. Consider the limit of both sides of (19) as $\Delta \rightarrow 0$ : if

$$
[\sigma-\beta(t)]\left[r_{B} q(t)+r_{S}(1-q(t))+\rho\right]<\rho \sigma
$$

then (19) must also hold for $\Delta>0$ sufficiently close to zero. The latter, however, follows because as $\Delta \rightarrow 0$, the final moment of settlement $m$ converges to $\tilde{m}$ and price $q_{m}$ converges to $\beta(\tilde{m})=$ $\left(r_{B}+r_{S}\right) \sigma /\left(r_{B}+r_{S}+\rho\right)$, hence $[\sigma-\beta(\tilde{m})]\left(r_{B}+r_{S}+\rho\right)=\rho \sigma$. Then, by $q(t) \in(0,1)$, inequality

$$
[\sigma-\beta(\tilde{m})]\left[r_{B} q(t)+r_{S}(1-q(t))+\rho\right]<\rho \sigma
$$

follows, hence the same is also true for $m$ replacing $\tilde{m}$ in this inequality provided $m$ is sufficiently close to $\tilde{m}$ (which it is, for $\Delta>0$ sufficiently small). But then, for all $t<m, \beta(t)>\beta(m)$, hence (20) also holds. This completes the proof.

Proof of Proposition 5. The fact that $\tilde{m}$ is increasing in $\sigma$ and $\rho$ but decreasing in $r_{B}$ and $r_{S}$ follow simply by inspecting (14), as mentioned in the main text.

In the rest of the proof assume $r_{B}=r_{S} \equiv r$. Recall that the settlement price at the final settlement date is $q(\tilde{m})=\beta(\tilde{m})=\sigma \lambda$, which is increasing in $\sigma$ and decreasing in $\rho$ because $\lambda=2 r /(2 r+\rho)$. By (15), the slope of the settlement price is

$$
\dot{q}(t)=(\rho+r)[q(t)-\beta(t)],
$$

which is zero at $t=\tilde{m}$ and negative for all $t<\tilde{m}$, where $q(t)<\beta(t)$.

For clarity (in this paragraph only), denote the settlement price at $t$ under prior $\sigma$ by $q(t \mid \sigma)$ and the final settlement date by $\tilde{m}_{\sigma}$. Let $\sigma^{\prime}>\sigma$. Now $\tilde{m}_{\sigma^{\prime}}>\tilde{m}_{\sigma}$, moreover,

$$
q\left(\tilde{m}_{\sigma} \mid \sigma^{\prime}\right)>q\left(\tilde{m}_{\sigma^{\prime}} \mid \sigma^{\prime}\right) \equiv \sigma^{\prime} \lambda>\sigma \lambda \equiv q\left(\tilde{m}_{\sigma} \mid \sigma\right)
$$

where the first inequality follows by $\dot{q}\left(t \mid \sigma^{\prime}\right)<0$ for $t<\tilde{m}_{\sigma^{\prime}}$ and $\tilde{m}_{\sigma}<\tilde{m}_{\sigma^{\prime}}$. Suppose, towards contradiction, that $q\left(t \mid \sigma^{\prime}\right)=q(t \mid \sigma)$ for some $t<\tilde{m}_{\sigma}$. But $\dot{q}(t \mid \sigma)$ is decreasing in the level of $\beta(t) \equiv$ $\sigma /\left[\sigma+(1-\sigma) e^{r_{S} t}\right]$, which is itself increasing in $\sigma$; hence $\dot{q}(t \mid \sigma)$ is decreasing in $\sigma$, holding the level of $q(t \mid \sigma)$ fixed. Therefore $\dot{q}\left(t \mid \sigma^{\prime}\right)<\dot{q}(t \mid \sigma)$, which contradicts $q\left(\tilde{m}_{\sigma} \mid \sigma^{\prime}\right)>q\left(\tilde{m}_{\sigma} \mid \sigma\right)$. Therefore $q\left(t \mid \sigma^{\prime}\right)>q(t \mid \sigma)$ for all $t<\tilde{m}_{\sigma^{\prime}}$, as claimed.

If $\rho=0$ then the seller skims with $p(t)=1$ for all $t>0$; the value of the good is eventually revealed by either player so the expected present value of his payoff is the same as if he could settle at price $\sigma$ at time 0 (which he cannot because $t \in\{\Delta, 2 \Delta, \ldots\}$ ). For small $\rho>0$ the final settlement price is 
$\beta(\tilde{m})<\sigma$, at $\tilde{m} \approx \rho / r$, which is obtained by Taylor expansion of (14) around $\rho=0$. The immediate settlement price, $q(\underline{t})$ is less than $\sigma$ as well, provided $\rho>0$ is sufficiently small, since $\dot{q}(\tilde{m})=0$.

\section{REFERENCES}

ABreU, D., AND F. Gul (2000): “Bargaining and Reputation,” Econometrica, 68(1), 85-117.

Admati, A. R., AND M. Perry (1987): "Strategic Delay in Bargaining," The Review of Economic Studies, 54(3), 345-364.

Ali, S. N. M. (2006): "Waiting to Settle: Multilateral Bargaining with Subjective Biases," Journal of Economic Theory, 130(1), 109-137.

AumanN, R. J., AND S. HART (2003): “Long Cheap Talk,” Econometrica, 71(6), 1619-1660.

Ausubel, L. M., P. CRAmton, AND R. J. DeneCKere (2002): “Bargaining with Incomplete Information," in Handbook of Game Theory with Economic Applications, ed. by R. J. Aumann, and S. Hart, vol. 3, chap. 50, pp. 1897-1945. North-Holland, Amsterdam.

BoARD, S., AND M. PyCiA (2014): "Outside Options and the Failure of the Coase Conjecture," American Economic Review, 104(2), 656-671.

BulOW, J. I. (1982): “Durable-Goods Monopolists," Journal of Political Economy, 90(2), 314-332.

DAHL, R. (1979): "Parson's Pleasure," in Tales of the Unexpected, pp. 193-217. Penguin, London.

Daley, B., AND B. GReEN (2012): "Waiting for News in the Market for Lemons," Econometrica, 80(4), 1433-1504.

- (2016): "Bargaining and News," Duke University and UC Berkeley, unpublished manuscript.

DEKEL, E. (1990): "Simultaneous Offers and the Inefficiency of Bargaining: A Two-Period Example," Journal of Economic Theory, 50(2), 300-308.

DRANOVE, D., AND G. Z. JIN (2010): "Quality Disclosure and Certification: Theory and Practice," Journal of Economic Literature, 48(4), 935-963.

Eső, P., AND C. WALlaCE (2014): "Information and Evidence in Bargaining," Economic Theory Bulletin, 2(1), 23-32.

FERSHTMAN, C., AND D. J. SEIDMANN (1993): “Deadline Effects and Inefficient Delay in Bargaining with Endogenous Commitment," Journal of Economic Theory, 60(2), 306-321.

FISHER, R., AND W. URY (1982): Getting to Yes: Negotiating an Agreement without Giving In. Random House, London.

Forges, F., AND F. KOESSLER (2008): “Long Persuasion Games,” Journal of Economic Theory, 143(1), 1-35.

FuchS, W., AND A. SKRZYPACZ (2010): “Bargaining with Arrival of New Traders," American Economic Review, 100(3), 802-836.

(2013): “Bargaining with Deadlines and Private Information," American Economic Journal: Microeconomics, 5(4), 219-243.

FudenberG, D., D. K. Levine, And J. Tirole (1985): “Infinite Horizon Models of Bargaining with One-Sided Incomplete Information," in Game-Theoretic Models of Bargaining, ed. by A. E. Roth, chap. 5, pp. 73-98. Cambridge University Press, Cambridge.

FudenberG, D., And J. Tirole (1991): Game Theory. MIT Press, Cambridge, MA.

Grossman, S. J. (1981): "The Informational Role of Warranties and Private Disclosure about Product Quality," Journal of Law and Economics, 24(3), 461-483.

Gul, F., H. SONNENSCHEIN, AND R. Wilson (1986): “Foundations of Dynamic Monopoly and the Coase Conjecture," Journal of Economic Theory, 39(1), 155-190.

HART, O. D., AND J. TiRole (1988): “Contract Renegotiation and Coasian Dynamics," The Review of Economic Studies, 55(4), 509-540. 
HÖRNER, J., AND A. SKRZYPACZ (2016): “Selling Information," Journal of Political Economy, 124(6), $1515-1562$.

HWANG, I., AND F. LI (2017): “Transparency of Outside Options in Bargaining," Journal of Economic Theory, 167, 116-147.

KamenicA, E., And M. Gentzkow (2011): "Bayesian Persuasion," American Economic Review, 101(6), 2590-2615.

KENNAN, J., AND R. WILSON (1993): “Bargaining with Private Information," Journal of Economic Literature, 31(1), 45-104.

Koessler, F., AND R. Renault (2012): “When does a Firm Disclose Product Information?," RAND Journal of Economics, 43(4), 630-649.

KOESSLER, F., AND V. SKRETA (2016): “Informed Seller with Taste Heterogeneity," Journal of Economic Theory, $165,456-471$.

(2017): "Selling with Evidence," Paris School of Economics and UCL, unpublished manuscript.

MATHIS, J. (2008): “Full Revelation of Information in Sender-Receiver Games of Persuasion," Journal of Economic Theory, 143(1), 571-584.

Milgrom, P. R. (1981): “Good News and Bad News," Bell Journal of Economics, 12(2), 380-391.

(2008): "What the Seller Won't Tell You: Persuasion and Disclosure in Markets," Journal of Economic Perspectives, 22(2), 115-131.

Milgrom, P. R., AND N. L. STOKey (1982): “Information, Trade and Common Knowledge," Journal of Economic Theory, 26(1), 17-27.

MuthoO, A. (1999): Bargaining Theory with Applications. Cambridge University Press, Cambridge. RaIfFA, H. (1982): The Art and Science of Negotiation. Harvard University Press, Cambridge, MA.

Rubinstein, A. (1982): “Perfect Equilibrium in a Bargaining Model," Econometrica, 50(1), 97-109.

SEIDMANN, D. J., AND E. WinTER (1997): "Strategic Information Transmission with Verifiable Messages," Econometrica, 65(1), 163-169.

Shavell, S. M. (1994): "Acquisition and Disclosure of Information Prior to Sale," RAND Journal of Economics, 25(1), 20-36.

SHER, I., AND R. VOHRA (2015): “Price Discrimination through Communication," Theoretical Economics, 10(2), 597-648.

SHIN, H. S. (1994): "The Burden of Proof in a Game of Persuasion," Journal of Economic Theory, 64(1), 253-264.

(2003): “Disclosures and Asset Returns," Econometrica, 71(1), 105-133.

Sobel, J., AND I. TAKAHASHI (1983): "A Multistage Model of Bargaining," The Review of Economic Studies, 50(3), 411-426.

STÅHL, I. (1972): Bargaining Theory. Stockholm School of Economics, Stockholm.

StOKey, N. L. (1981): "Rational Expectations and Durable Goods Pricing," The Bell Journal of Economics, 12(1), 112-128.

TYSON, C. J. (2010): “Dominance Solvability of Dynamic Bargaining Games," Economic Theory, 43(3), 457-477.

YILDIZ, M. (2003): "Bargaining without a Common Prior-An Immediate Agreement Theorem," Econometrica, 71(3), 793-811.

(2004): “Waiting to Persuade," The Quarterly Journal of Economics, 119(1), 223-248. 